\title{
ALMA Polarimetry of Sgr A*: Probing the Accretion Flow from the Event Horizon to the Bondi Radius
}

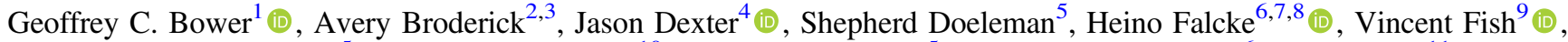 \\ Michael D. Johnson ${ }^{5}\left(\mathbb{0}\right.$, Daniel P. Marrone ${ }^{10}{ }^{(1)}$, James M. Moran ${ }^{5}$, Monika Moscibrodzka ${ }^{6}$, Alison Peck ${ }^{11} \mathbb{D}^{1}$, \\ Richard L. Plambeck ${ }^{12}$ (D), and Ramprasad Rao ${ }^{1}$ (DD \\ ${ }^{1}$ Academia Sinica Institute of Astronomy and Astrophysics, 645 N. A’ohoku Place, Hilo, HI 96720, USA; gbower@asiaa.sinica.edu.tw \\ ${ }^{2}$ Department of Physics and Astronomy, University of Waterloo, 200 University Avenue West, Waterloo, ON, N2L 3G1, Canada \\ ${ }^{3}$ Perimeter Institute for Theoretical Physics, 31 Caroline Street North, Waterloo, ON, N2L 2Y5, Canada \\ ${ }_{5}^{4}$ Max Planck Institute for Extraterrestrial Physics, Giessenbachstr. 1, D-85748 Garching, Germany \\ ${ }^{5}$ Harvard-Smithsonian Center for Astrophysics, 60 Garden Street, Cambridge, MA 02138, USA \\ ${ }^{6}$ Department of Astrophysics, Institute for Mathematics, Astrophysics and Particle Physics (IMAPP), Radboud University, P.O. Box 9010, \\ 6500 GL Nijmegen, The Netherlands \\ ${ }^{7}$ ASTRON, P.O. Box 2, 7990 AA Dwingeloo, The Netherlands \\ ${ }^{8}$ Max-Planck-Institut für Radioastronomie, Auf dem Hügel 69, D-53121 Bonn, Germany \\ ${ }^{9}$ Massachusetts Institute of Technology, Haystack Observatory, Route 40, Westford, MA 01886, USA \\ ${ }^{10}$ Steward Observatory, University of Arizona, 933 North Cherry Avenue, Tucson, AZ 85721, USA \\ ${ }^{11}$ Gemini Observatory, 670 N. A'ohoku Pl., Hilo, HI 96720, USA \\ ${ }^{12}$ Radio Astronomy Laboratory, University of California, Berkeley, CA 94720-3411, USA \\ Received 2018 August 30; revised 2018 October 15; accepted 2018 October 16; published 2018 November 27
}

\begin{abstract}
Millimeter polarimetry of Sgr $\mathrm{A}^{*}$ probes the linearly polarized emission region on a scale of $\sim 10$ Schwarzschild radii $\left(R_{S}\right)$, as well as the dense, magnetized accretion flow on scales out to the Bondi radius $\left(\sim 10^{5} R_{S}\right)$ through Faraday rotation. We present here multi-epoch ALMA Band $6(230 \mathrm{GHz})$ polarimetry of Sgr A*. The results confirm a mean rotation measure, $\mathrm{RM} \approx-5 \times 10^{5} \mathrm{rad} \mathrm{m}^{-2}$, consistent with measurements over the past $20 \mathrm{yr}$, and support an interpretation of the RM as originating from a radiatively inefficient accretion flow with $\dot{M} \approx 10^{-8} M_{\odot} \mathrm{yr}^{-1}$. Variability is observed for the first time in the RM on timescales that range from hours to months. The long-term variations may be the result of changes in the line-of-sight properties in a turbulent accretion flow. Short-term variations in the apparent RM are not necessarily the result of Faraday rotation and may be the result of complex emission and propagatation effects close to the black hole, some of which have been predicted in numerical modeling. We also confirm the detection of circular polarization at a mean value of $-1.1 \% \pm 0.2 \%$. It is variable in amplitude on timescales from hours to months, but the handedness remains unchanged from that observed in past centimeter- and millimeter-wavelength detections. These results provide critical constraints for the analysis and interpretation of Event Horizon Telescope data of Sgr A*, M87, and similar sources.
\end{abstract}

Key words: accretion, accretion disks - black hole physics - galaxies: jets - galaxies: nuclei Galaxy: center - polarization

\section{Introduction}

Sgr $\mathrm{A}^{*}$ is the $\sim 4.1 \times 10^{6} M_{\odot}$ black hole in the Galactic center (Falcke \& Markoff 2013; Boehle et al. 2016; Gravity Collaboration et al. 2018). As the nearest supermassive black hole, it serves as a powerful laboratory for the understanding of accretion, outflow, and jet physics, as well as detailed physics associated with particle acceleration and magnetic fields (Ressler et al. 2017; Davelaar et al. 2018). Sgr $\mathrm{A}^{*}$ is also an important target for tests of general relativity and measurement of intrinsic black hole parameters such as spin and the presence of the event horizon through a variety of approaches including measurement of stellar orbits (Waisberg et al. 2018) and imaging of event horizon scale structure (Falcke et al. 2000; Broderick et al. 2014). Convincing tests of GR and characterization of black hole properties through imaging rely on a thorough and detailed understanding of accretion, outflow, and particle acceleration physics.

Imaging of $\mathrm{Sgr}^{*}{ }^{*}$ at millimeter and submillimeter wavelengths is a major goal of the Event Horizon Telescope (Doeleman et al. 2009; Fish et al. 2013). EHT observations will have an angular resolution comparable to the Schwarzschild radius $\left(1 R_{S} \approx 10 \mu a s\right.$ for a distance of $\left.8.1 \mathrm{kpc}\right)$ and are sensitive to structures on scales as large as a few $\times 10 R_{S}$. Imaging has established the dominance of angular broadening due to scattering by interstellar electrons along the line of sight (Bower et al. 2006) and an intrinsic source size that is $\sim 10 R_{S}$ at $3 \mathrm{~mm}$ wavelength (Bower et al. 2004; Shen et al. 2005; Bower et al. 2014; Brinkerink et al. 2016; Ortiz-León et al. 2016) and $\sim 4 R_{S}$ at $1.3 \mathrm{~mm}$ wavelength (Doeleman et al. 2008; Fish et al. 2011; Johnson et al. 2015; Fish et al. 2016; Lu et al. 2018). These radio and millimeter/submillimeter results cannot be conclusively interpreted in terms of either accretion disk or jet models, leaving the question whether a jet is present unanswered. Images obtained with the EHT will be sensitive to the accretion flow and/or jet-launching region on scales of a few $R_{S}$ (Mościbrodzka et al. 2014).

The region imaged by the EHT is embedded within the larger accretion flow of Sgr A*. Chandra X-ray imaging shows an extended structure with a scale comparable to the Bondi radius, $\sim 10^{5} R_{S}$ (Wang et al. 2013). The accretion rate at the Bondi radius is estimated to be $\sim 10^{-4}-10^{-5} M_{\odot} \mathrm{yr}^{-1}$ and fed by stellar winds of massive stars outside the accretion flow (Quataert et al. 1999). This accretion rate appears to be inconsistent with Bondi accretion 
onto the black hole, producing the very low bolometric luminosity $L_{\text {bol }} \sim 10^{35} \mathrm{erg} \mathrm{s}^{-1}$ of Sgr A*. This has driven the development of a number of theoretical models that fall under the umbrella of radiatively inefficient accretion flows (RIAFs; Yuan \& Narayan 2014). Broadly, these models produce the low luminosity of Sgr A* through two mechanisms: stalled accretion at large radii, which reduces the accretion rate, and two-temperature plasmas in which the lower-temperature electrons do not equilibrate with the full gravitational potential energy of infall and, therefore, radiate a small fraction of the total available energy.

Millimeter- and submillimeter-wavelength polarimetry of Sgr A* has been a powerful tool for characterization of the accretion flow on scales inside the Bondi radius that are inaccessible to other techniques. Sgr A* shows linear polarization (LP) and circular polarization (CP) properties that are not common in higher-power active galactic nuclei (AGNs). Sgr A* shows no LP at centimeter wavelengths (Bower et al. 1999a, 1999c), while at millimeter/submillimeter wavelengths the polarization fraction rises to $\sim 10 \%$ (Aitken et al. 2000; Bower et al. 2003; Macquart et al. 2006; Marrone et al. 2007; Liu et al. 2016a, 2016b). The LP has been shown to undergo significant Faraday rotation, with one of the largest rotation measures (RMs) observed in any source, $\mathrm{RM} \approx-5 \times 10^{5} \mathrm{rad} \mathrm{m}^{-2}$. The $\mathrm{RM}$ is proportional to the line-of-sight integrated electron density and parallel magnetic field strength:

$$
\mathrm{RM}=0.81 \int n_{e} \boldsymbol{B} \cdot \boldsymbol{d} \boldsymbol{s} \mathrm{rad} \mathrm{\textrm {m } ^ { - 2 }}
$$

where $n_{e}$ is in units of $\mathrm{cm}^{-3}, \boldsymbol{B}$ is in $\mu \mathrm{G}$, and the length scale is in pc. The LP properties have been interpreted as intrinsic polarization arising within $\sim 10 R_{S}$ of the event horizon and propagating through the dense, magnetized accretion flow. The $\mathrm{RM}=-7 \times 10^{4} \mathrm{rad} \mathrm{m}^{-2}$ found for the GC pulsar J1745-2900, which is separated by $\sim 0.1$ pc from Sgr A*, supports the hypothesis that the majority of the Sgr A* RM originates in the accretion flow (Eatough et al. 2013; Bower et al. 2015a). Monitoring of the pulsar RM also demonstrates that interstellar medium (ISM) changes in the RM are $\sim 10^{4} \mathrm{rad} \mathrm{m}^{-2}$, significant for a pulsar, but small relative to the Sgr A* RM (Desvignes et al. 2018). In the accretion flow interpretation for Sgr $A^{*}$, the RM demonstrates a profile for the electron density as a function of radius (e.g., $n_{e} \propto r^{-1}$ ) that is flatter than required by advectiondominated accretion flow (ADAF) models and sets an accretion rate onto $\mathrm{Sgr} \mathrm{A}^{*}$ at the event horizon of $\sim 10^{-8} M_{\odot} \mathrm{yr}^{-1}$.

The $1.3 \mathrm{~mm}$ wavelength very long baseline interferometry (VLBI) supports the conclusion that the LP originates within $\sim 10 R_{S}$ (Johnson et al. 2015). These observations show that the LP does not originate from a simple, homogeneous source but from a more complex source with structure in the magnetic field (and, hence, polarization angle) on scales of $\lesssim 10 R_{S}$. Complex polarization features are predicted in general relativistic magnetohydrodynamical (GRMHD) models of Sgr A* accretion disks and jets (Shcherbakov et al. 2012; Gold et al. 2017; Mościbrodzka et al. 2017).

$\mathrm{CP}$ is present in $\mathrm{Sgr} \mathrm{A}^{*}$ from centimeter to submillimeter wavelengths, a factor of more than 200 in wavelength (Bower et al. 1999b; Sault \& Macquart 1999; Bower et al. 2002b; Muñoz et al. 2012). The CP has constant handedness across all wavelengths and a magnitude $\lesssim 1 \%$. The origin of the CP is not well understood, but it is unlikely to be produced through the synchrotron mechanism. It is more likely that the process of
Table 1

ALMA Observations

\begin{tabular}{lc}
\hline \hline Epoch & UT \\
\hline 2016 Mar 3 & $0936-1314$ \\
2016 May 3 & $0537-0913$ \\
2016 Aug 13 & $2057-0357$ \\
\hline
\end{tabular}

Faraday conversion (Pacholczyk 1977) transforms LP into CP via thermal electrons that are mixed with the relativistic electrons responsible for the linearly polarized synchrotron emission (Beckert \& Falcke 2002; Ruszkowski \& Begelman 2002; Huang et al. 2008). The stability of the handedness of the $\mathrm{CP}$ over decades suggests a stable magnetic field configuration in the emission and conversion region.

Time variablity of the polarization properties has long been recognized as an important diagnostic of the accretion flow properties (Bower et al. 2005; Marrone et al. 2007). The timescale of variability for the RM can be translated into a radius at which the $\mathrm{RM}$ originates, a kind of Faraday tomography. The orbital period at the innermost stable circular orbit is $\sim 30$ minutes, while the orbital period at the Bondi radius is $\sim 10^{3}$ yr. Characterization of the RM variability over timescales up to $10 \mathrm{yr}$ provides sensitivity to radii as large as $10^{3} R_{S}$, much larger than can be probed through submillimeter VLBI imaging and much smaller than can be probed through direct X-ray imaging of the accretion flow. A variety of models of turbulence and magnetic field structure in the accretion flow predict different degrees of RM variability (Sharma et al. 2007; Pang et al. 2011). GRMHD models are now explicitly modeling variations in polarization properties, including Faraday effects on scales as small as the emission region (Mościbrodzka et al. 2017).

Sgr $\mathrm{A}^{*}$ is not alone in showing these unusual polarization properties. Three other low-luminosity AGNs (LLAGNs) have now been demonstrated to have large RMs: M87 (Kuo et al. 2014), 3C 84 (Plambeck et al. 2014), and 3C 273 (Hovatta et al. 2018). A number of other LLAGNs appear to have suppressed LP at centimeter and millimeter wavelengths, possibly as the result of extreme RMs (Brunthaler et al. 2001; Bower et al. 2002a, 2017).

The sensitivity and systematic control of the Atacama Large Millimeter Array (ALMA) provides a powerful tool for detailed characterization of the LP, CP, and Faraday properties of Sgr A*. We present here new full-Stokes observations obtained via ALMA Cycle 2. In Section 2, we present the observations and data reduction. In Section 3, we present our results. Given the novelty of ALMA polarimetry, we place significant emphasis on validation of the results through examination of calibrator sources in Appendix A. In Section 4, we discuss these results and their implications for accretion and outflow models of Sgr A*. We give our conclusions in Section 5.

\section{Observations}

ALMA observed Sgr A* in Band 6 (1.3 mm wavelength) in full polarimetric continuum mode on three epochs (Table 1). The correlator was configured with four spectral windows (SPWs), each with $2 \mathrm{GHz}$ bandwidth in 64 channels. The SPW center frequencies are 223.96, 225.96, 239.96, and $241.96 \mathrm{GHz}$. The Band 6 receivers are sensitive to LP (X and $Y$ ), and the correlator produces $X X, Y Y, X Y$, and $Y X$ 
correlations. The array was in a relatively compact configuration with naturally weighted synthesized beam sizes of $\sim 1^{\prime \prime}$.

Sources were observed for amplitude, bandpass, and polarization calibration $(\mathrm{J} 1751+0939)$ and for phase calibration (J1733-3722). Absolute flux calibration was set by observations of the moon Titan in epochs 1 and 2. In epoch 3 , absolute flux calibration was set by ALMA monitoring of $\mathrm{J} 1751+0939$ at 90 and $345 \mathrm{GHz}$; a power-law extrapolation to $230 \mathrm{GHz}$ was used to provide the estimated flux density of $2.4 \mathrm{Jy}$, constant across all SPWs, with an accuracy of $\sim 20 \%$. A check source (J1713-3418) was interleaved with phase calibration and $\mathrm{Sgr} \mathrm{A}^{*}$ observations. In epoch 3, additional short observations were obtained on J1517-2422, J1924-2914, and J1733-1304. A typical observation cycle included $30 \mathrm{~s}$ on the phase calibrator, $20 \mathrm{~s}$ on the check source, and 7 minutes on Sgr A*. Approximately $3 \mathrm{hr}$ of observations were obtained in epochs 1 and 2 . Approximately $5 \mathrm{hr}$ of observations were obtained over the $7 \mathrm{hr}$ duration of epoch 3 owing to failure to observe two hour-long observing sequences known as execution blocks.

Data reduction was performed in CASA using pipelines that applied standard a priori and calculated calibrations. This produced calibrated measurement sets with the full time and frequency resolution of the observations. We extracted source data by imaging with all of the data averaged over all channels and various subsets of the data, sliced in frequency and in time. We rejected all baselines shorter than $50 k \lambda$ in order to eliminate any extended structure around Sgr A*. For consistency, we applied the same baseline cut to all calibrator data. Results were obtained by fitting point sources in the image domain. Fits in the Stokes $Q, U$, and $V$ domains were obtained with the pointsource position fixed at the fitted peak of the Stokes $I$ image. These image domain results are consistent with point-source fits obtained in the visibility domain.

\section{Results}

In Appendix A, we present results for calibrators with the goal of demonstrating the stability of the ALMA polarization measurements and determination of systematic limits on polarization quantities. We find that results are most stable and accurate for sources observed at multiple parallactic angles. Inter-epoch results have limits for fractional LP and CP near $\sim 0.1 \%-0.2 \%$. Position angles are measured to an accuracy of $\sim 1^{\circ}$, and RMs are determined to an accuracy $\lesssim 10^{5} \mathrm{rad} \mathrm{m}^{-2}$. Intra-epoch measurements have similar accuracy. We caution that calibration errors will play a larger role the smaller the polarization fraction; all calibrators have a polarization fraction larger than $1 \%$. In this section, we present results for Sgr $\mathrm{A}^{*}$ in inter-epoch and intra-epoch measurements.

\subsection{Inter-epoch Polarization Properties of Sgr $A^{*}$}

In Table 2 we summarize the time-averaged polarization properties of Sgr $A^{*}$ in each epoch and for each SPW. Sgr A* is detected with high significance in each epoch, each SPW, and each Stokes parameter. We show SPW-averaged polarization position angle as a function of wavelength squared in the three epochs in Figure 1, revealing a clear variation in RM between these epochs. In Figure 2, we show the polarization position angle as a function of wavelength squared for each individual channel, with separate plots for each epoch. The results are consistent in a comparison between the SPWaveraged and channel-averaged presentations.

We fit the RM and the mean-wavelength $(\bar{\lambda})$ position angle $(\bar{\chi})$ following the relation

$$
\chi=\chi_{0}+\mathrm{RM} \lambda^{2}
$$

where $\chi$ is the observed position angle at wavelength $\lambda$ and $\chi_{0}$ is the position angle at zero wavelength. An RM of $1.1 \times 10^{7} \mathrm{rad} \mathrm{m}^{-2}$ corresponds to a full rotation of the position angle over the full frequency range. Thus, observations with $\mathrm{RM}>$ few $\times 10^{6} \mathrm{rad} \mathrm{m}^{-2}$ will have $\sim 1 \mathrm{rad}$ of phase wrap between the highest and lowest frequencies, which could lead to a phase-wrap ambiguity. All RM fitting in this paper is done using a weighted least-squares method of the position angle against $\lambda^{2}$. This method is suitable for the limited range of position angles typically present and the uniformity of errors in the data. In Table 3, we summarize the average RM fits to the data. These fits are also plotted in Figures 1 and 2 . The quality of these fits is consistent with no deviation from a $\lambda^{2}$ law for the position angle in the average polarization properties. The significance of the RM detection for $\mathrm{Sgr} \mathrm{A}^{*}$ is $\gtrsim 100 \sigma$ in each epoch. In fact, we find the goodness of fit $\chi_{\nu}^{2} \sim 10^{-3}$ for the fits to the SPW-averaged data (Figure 1), suggesting that we are significantly overestimating the errors in $Q$ and $U$ in our fitting. This is not surprising given the extremely high dynamic range of these images and the limited number of data points (four) contributing to an individual RM calculation. We calculate errors in the RM based on the scatter of the residual phases after fitting. For the individual channel results (Figure 2), we find $\chi_{\nu}^{2}=31,2.5$, and 0.4 for the three epochs, respectively. These results suggest that their could be some additional systematic contributions to the RM residual.

We also fit slopes $\delta$ to $I, V$, and $p$ as a function of frequency and summarize these in Table 3. These slopes test whether a nonzero spectral index is a reasonable fit to these data. For all of the sources we see marginal or no evidence for a Stokes $I$ spectral index change over the $18 \mathrm{GHz}$ range of these observations. In LP and $\mathrm{CP}, \delta p$ and $\delta V$ for $\mathrm{Sgr} \mathrm{A}^{*}$ are significant, change sign between epochs, and are an order of magnitude larger than for other calibrators observed for a full track.

\subsection{Intra-epoch Properties}

In Figures 3-5, we present intra-epoch light curves in Stokes $I, Q, U$, and $V$ for Sgr $\mathrm{A}^{*}$. Data are averaged over individual scans, which range from tens of seconds to 7 minutes in duration. We also show time-dependent fits of RM, $\chi_{0}$, and $p$ to the data for these same sources, as well as the residual position angle after the fits.

We see significant variability in all four Stokes parameters for Sgr $A^{*}$ within each epoch. We also see SPW-dependent variations in the Stokes parameters. The variations in RM and $\chi_{0}$ for Sgr $\mathrm{A}^{*}$ are an order of magnitude larger than those seen for the calibrator and check source (Appendix A). Position angle changes in the three epochs are $90^{\circ}, 25^{\circ}$, and $50^{\circ}$, respectively. Large intra-epoch polarization angle changes have been previously seen (Marrone et al. 2006; Johnson et al. 2015). Apparent RM variations are several times $10^{5} \mathrm{rad} \mathrm{m}^{-2}$ per epoch and as large as $10^{7} \mathrm{rad} \mathrm{m}^{-2}$ in epoch 1. The largest fitted values of the RM are suspect for three 
Table 2

Average Polarization Properties

\begin{tabular}{|c|c|c|c|c|c|c|}
\hline Source & Epoch & SPW & $\begin{array}{c}I \\
(\mathrm{mJy})\end{array}$ & $\underset{(\mathrm{mJy})}{Q}$ & $\begin{array}{c}U \\
(\mathrm{mJy})\end{array}$ & $\begin{array}{c}V \\
(\mathrm{mJy})\end{array}$ \\
\hline$\overline{\text { Sgr A* }}$ & 1 & 0 & $4062 \pm 130$ & $140.533 \pm 4.370$ & $79.584 \pm 2.486$ & $-50.173 \pm 1.618$ \\
\hline$\ldots$ & $\ldots$ & 1 & $3962 \pm 126$ & $132.145 \pm 4.038$ & $84.040 \pm 2.619$ & $-49.793 \pm 1.568$ \\
\hline$\ldots$ & $\ldots$ & 3 & $4110 \pm 126$ & $81.717 \pm 2.385$ & $107.850 \pm 3.195$ & $-55.053 \pm 1.730$ \\
\hline Sgr A* & 2 & 0 & $3435 \pm 68$ & $-176.558 \pm 3.425$ & $-190.601 \pm 3.795$ & $-30.207 \pm 0.667$ \\
\hline$\cdots$ & $\cdots$ & 3 & $3448 \pm 64$ & $-129.888 \pm 2.492$ & $-234.454 \pm 4.307$ & $-31.992 \pm 0.669$ \\
\hline$\overline{\text { Sgr } A^{*}}$ & 3 & 0 & $2657 \pm 82$ & $82.909 \pm 2.512$ & $-121.412 \pm 3.909$ & $-39.496 \pm 1.222$ \\
\hline$\cdots$ & $\ldots$ & 1 & $2582 \pm 80$ & $85.942 \pm 2.617$ & $-120.428 \pm 3.894$ & $-36.879 \pm 1.134$ \\
\hline$\ldots$ & $\ldots$ & 2 & $2735 \pm 85$ & $126.549 \pm 3.873$ & $-138.611 \pm 4.565$ & $-33.426 \pm 1.009$ \\
\hline$\ldots$ & $\ldots$ & 3 & $2741 \pm 86$ & $131.360 \pm 4.055$ & $-142.502 \pm 4.719$ & $-30.809 \pm 0.920$ \\
\hline
\end{tabular}

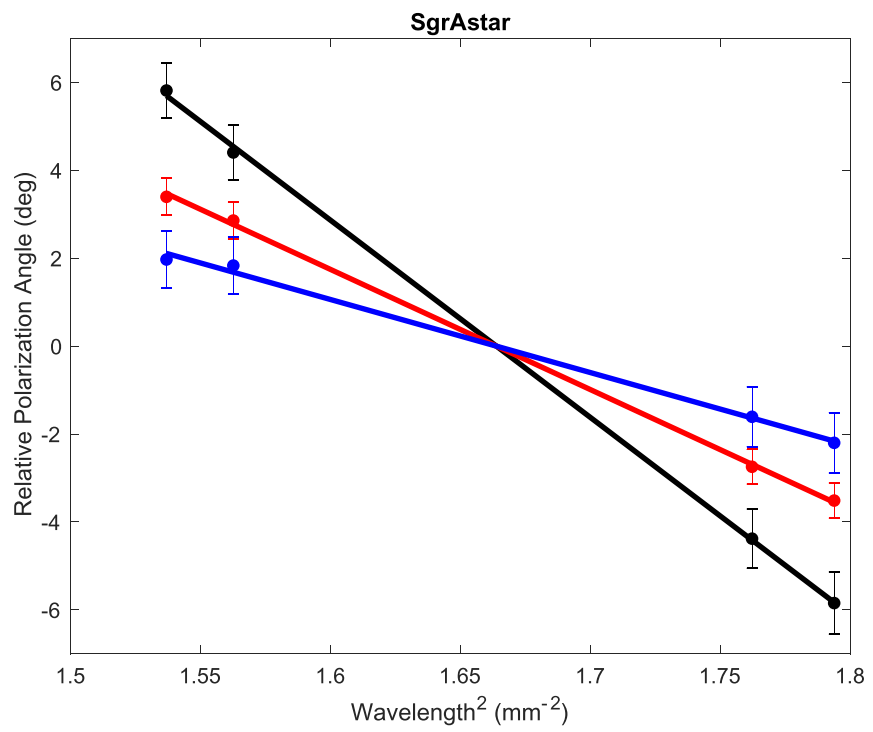

Figure 1. Average residual polarization position angle as a function of wavelength squared for Sgr $\mathrm{A}^{*}$ and each of the calibrators in epochs 1 (black), 2 (red), and 3 (blue). We have removed the mean position angle in each epoch to enable clear comparison.

reasons: (1) the phase-wrap ambiguity at $1.1 \times 10^{7} \mathrm{rad} \mathrm{m}^{-2}$; (2) the large error bars in the estimates and the large residual phases, indicative of poor quality fits; and (3) the low polarization fraction at the time of these measurements, placing our analysis in a regime where calibration errors can have a larger effect. We calculate the goodness-of-fit statistic $\chi_{\nu}^{2}$ for the results presented in Figure 3-5. For epochs 2 and 3, $\chi_{\nu}^{2} \approx 0.1$ at all times. For epoch $1, \chi_{\nu}^{2} \lesssim 1$ up to approximately 12:15 UT. After this time, $\chi_{\nu}^{2} \gg 1$. The small values of $\chi_{\nu}^{2}$ in epochs 2 and 3 and the beginning of epoch 1 are consistent with excellent fits to standard Faraday rotation with a modest overestimate of errors at some of the time. The large scatter in $\delta \chi_{0}$ in the latter half of epoch 1 reflects the time in which the $\chi_{\nu}^{2} \gg 1$. These poor fits come at times when the polarization fraction is the lowest.

We do not explore in detail the time-variable properties of the RM determined through fitting of the highest-frequency resolution (64-channel per SPW) data. In Figures 6-8, we show selected scans from each epoch at high-frequency resolution.
These results are consistent with the SPW-averaged results. That is, we find similar values of RM and $\chi_{0}$ from these data. Further, the slopes within each SPW are consistent with the slope between SPWs. In principle, these data can be searched for multiple RM components and/or non- $\lambda^{2}$ effects. However, the possibility for uncalibrated and time-variable systematic error in the highest-frequency resolution polarization data prevents us from making further use of these results. Nagai et al. (2016), for example, find frequency-dependent polarization leakage terms that, if not properly calibrated, could be misinterpreted as complex Faraday signatures. These highfrequency resolution plots give a clear indication of where non- $\lambda^{2}$ effects are most prominent, because the slopes within and between SPWs can be more directly compared. For instance, the fit in epoch 1 at 12:54 UT gives $|\mathrm{RM}|>10^{7} \mathrm{rad} \mathrm{m}^{-2}$, but clearly the slope between adjacent SPWs is consistent with a much smaller value of the RM. The fit at 12:56 UT shows similar properties but derives an $|\mathrm{RM}| \approx 10^{6} \mathrm{rad} \mathrm{m}^{-2}$ in part because of the phase wrap. In epoch 3, the fit at 01:24 UT also shows a broken slope, although the SPW-averaged fit at this time returns $\chi_{\nu}^{2}<1$.

\section{Analysis}

The results of these observations are qualitatively similar to previous measurements of Sgr A*, but they are significantly more accurate, which enables a more detailed picture of the polarization properties as a function of time and frequency. We obtain several significant results, which we explore in greater detail below: (1) polarized intensity variability on a timescale of months, with a variable spectral index; (2) confirmation of the presence of $\mathrm{CP}$ and detection of variability on a timescale of months; (3) variability in the LP and CP on a timescale of hours; (4) variability in the RM on a timescale of months, while remaining consistent with the long-term average and sign; and (5) short-term variability in the RM, which is coupled with changes in the polarized intensity and position angle.

The agreement in mean RM with the historical value and the small variations in the epoch-averaged properties strongly support the interpretation of Faraday rotation arising in the accretion flow with contributions dominating at radii $\left(10^{3}-10^{5}\right) R_{S}$. Under the RIAF interpretation, we find consistency with a constraint of $\dot{M} \sim 10^{-8} M_{\odot} \mathrm{yr}^{-1}$ (Marrone et al. 2007). The uncertainty in $\dot{M}$ arises from assumptions 

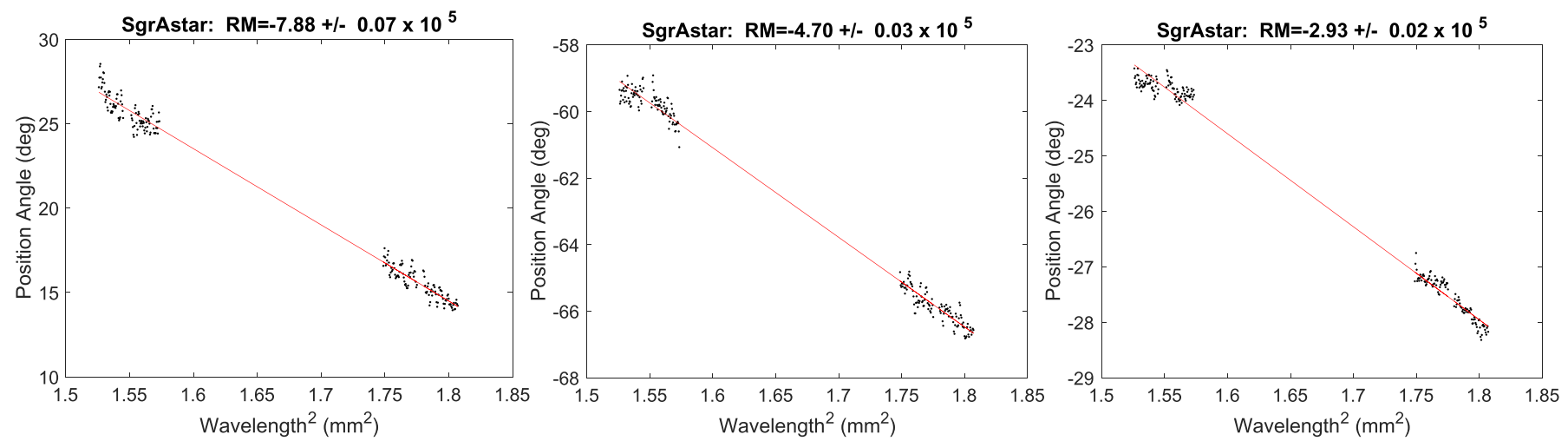

Figure 2. Polarization angle as a function of wavelength squared, presented for each channel and for each epoch. Epochs 1, 2, and 3 are shown in the left, middle, and right panels, respectively. Note the different scales for position angle. The title gives the RM in units of $\mathrm{rad} \mathrm{m}^{-2}$.

about the accretion flow model, rather than measurement uncertainty.

\subsection{Average Polarized Intensity}

The mean LP between epochs ranges from 3.6\% to $7.8 \%$, consistent with previous measurements (Aitken et al. 2000; Bower et al. 2003; Macquart et al. 2006; Marrone et al. 2007). The degree of LP appears to be independent of the total intensity per epoch. The total intensity has a flat spectrum across our frequency range. The polarized intensity, on the other hand, shows evidence for a slope across the band, corresponding to a change of $\lesssim 20 \%$ with a time-variable sign. Analyses of unpublished SMA and CARMA data suggest that there may be a preferred position angle at zero wavelength, $\chi_{0} \sim 180^{\circ}$. Epochs 2 and 3 show similar values for $\chi_{0}$, but epoch 1 has a mean value $\chi_{0}=95.3 \pm 0.9$ (Table 3 and Figure 9). We conclude that there is preferred intrinsic position angle, near $180^{\circ}$, but there is substantial variability in the intrinsic position angle.

We find a mean $\mathrm{CP}$ of $\approx-1.1 \% \pm 0.2 \%$. We find a change in the CP across the band that is as large as $25 \%$ in the third epoch. The sign of the frequency-dependent slope is time variable. We note that for the three epochs there is a linear correlation between $\delta P$ and $\delta V$, but there is no clear connection between $P$ and $V$. There is also no apparent relationship between either of the polarized and total intensity quantities.

We confirm the SMA detection of $\mathrm{CP}$ with a value of $-1.2 \% \pm 0.3 \%$ at $1.3 \mathrm{~mm}$ and $-1.6 \% \pm 0.4 \%$ at $0.86 \mathrm{~mm}$ (Muñoz et al. 2012). The results also suggest that the handedness of the millimeter-wavelength $\mathrm{CP}$ is stable on timescales greater than the $11 \mathrm{yr}$ span between the earliest SMA observation and the latest ALMA observation. This stability mirrors that of the centimeter-wavelength $\mathrm{CP}$, which has been shown to be stable for greater than 20 years (Bower et al. 2002b). If the centimeter- and millimeter-wavelength CPs originate via the same mechanism, then the handedness of that mechanism is apparently stable over almost $40 \mathrm{yr}$.

The LP is best explained through an origin in synchrotron emission close to the event horizon. Muñoz et al. (2012) provide a detailed discussion of potential origins for the $\mathrm{CP}$ emission. Faraday conversion is the favored mechanism for the production of $\mathrm{CP}$ in which thermal electrons are cospatial with the relativistic synchrotron-emitting electrons. A coherent magnetic field on the scale of the $\tau=1$ surface is required to produce Faraday conversion and the stable sign of the $\mathrm{CP}$ seen at all wavelengths. In a uniform medium, the sign of $\mathrm{CP}$ is expected to alternate as the phase shift between two LPs is $\propto n_{e} B^{2} \lambda^{3}$. This leads to frequent reversals at long wavelengths. Alternatively, for a stratified synchrotron source an appropriate scaling of the electron density and magnetic field with radius can counter the wavelength dependence and lead to an apparently flat spectrum. We then require a stable magnetic field geometry on scales of the source size, which ranges from a few $R_{S}$ to hundreds or thousands of $R_{S}$. One specific model for achieving the proper stratification is through magnetic field shear in the accretion flow (A. Broderick et al. 2018, in preparation).

\subsection{Rotation Measure in Multiple Epochs}

We show clearly that the RM has varied across the three epochs of our observations. Even with a conservative estimate of a random systematic error per epoch of $10^{5} \mathrm{rad} \mathrm{m}^{-2}$, the change $\delta \mathrm{RM}=4.93 \times 10^{5} \mathrm{rad} \mathrm{m}^{-2}$ across the three epochs is detected at a $5 \sigma$ threshold. Using only the thermal error, the change has a significance of $>30 \sigma$.

In Table 4 and Figure 10, we summarize published historical measurements of the RM. The RM values that we find fall within the bounds of previous variations. But none of these previous variations could be determined to be significant given the lower signal-to-noise ratio of detection and small lever arms. Additional unpublished RMs obtained with CARMA and SMA in intervening years fall within the same range.

We analyze variability in the RM using the structure function

$$
\operatorname{SF}^{2}(\tau)=\left\langle(\operatorname{RM}(t+\tau)-\operatorname{RM}(t))^{2}\right\rangle .
$$

The structure function calculates the characteristic variability on a timescale $\tau$. While the structure function has some known limitations in accurately determining saturation timescales (Emmanoulopoulos et al. 2010), it is suitable for our purposes. In Figure 11, we show the SF calculated for each epoch individually and for all of the epoch-averaged results, archival and current. Our data are too sparsely sampled to achieve the statistical ensemble average implied by Equation (3). Errors are determined from the scatter of RM differences measured within a time bin. Hence, the error is not defined when we calculate the SF for the ALMA inter-epoch timescales; these points should be treated as instances to be included in the larger ensemble. Further, we cannot properly address uncertainty in the SF on the longest timescales since we are only capturing a 
Table 3

Frequency-averaged Polarization and Rotation Measures

\begin{tabular}{|c|c|c|c|c|c|c|c|c|c|}
\hline Source & Epoch & $\begin{array}{c}I \\
(\mathrm{mJy})\end{array}$ & $\begin{array}{c}\delta I \\
\left(\mathrm{mJy} \mathrm{GHz}^{-1}\right)\end{array}$ & $\begin{array}{c}p \\
(\mathrm{mJy})\end{array}$ & $\begin{array}{c}\delta p \\
\left(\mathrm{mJy} \mathrm{GHz}^{-1}\right)\end{array}$ & $\begin{array}{c}V \\
(\mathrm{mJy})\end{array}$ & $\begin{array}{c}\delta V \\
\left(\mathrm{mJy} \mathrm{GHz}^{-1}\right)\end{array}$ & $\begin{array}{c}\mathrm{RM} \\
\left(10^{5} \mathrm{rad} \mathrm{m}^{-2}\right)\end{array}$ & $\begin{array}{c}\chi_{0} \\
(\operatorname{deg})\end{array}$ \\
\hline Sgr A* $^{*}$ & 1 & $4051 \pm 28$ & $4.8 \pm 28.1$ & $147.92 \pm 0.48$ & $-1.39 \pm 0.06$ & $-52.309 \pm 0.253$ & $-0.290 \pm 0.032$ & $-7.83 \pm 0.10$ & $95.3 \pm 0.9$ \\
\hline$\cdots$ & 2 & $3415 \pm 23$ & $2.8 \pm 22.5$ & $261.51 \pm 1.85$ & $0.56 \pm 0.23$ & $-31.576 \pm 0.742$ & $-0.186 \pm 0.092$ & $-4.77 \pm 0.07$ & $-17.4 \pm 0.7$ \\
\hline$\ldots$ & 3 & $2678 \pm 23$ & $7.1 \pm 23.2$ & $169.07 \pm 1.26$ & $2.68 \pm 0.16$ & $-35.100 \pm 0.663$ & $0.395 \pm 0.082$ & $-2.90 \pm 0.11$ & $2.0 \pm 1.1$ \\
\hline
\end{tabular}

Table 4

Historical Measurements of Rotation Measure

\begin{tabular}{lcc}
\hline \hline Source & Date & $\begin{array}{c}\mathrm{RM} \\
\left(10^{5} \mathrm{rad} \mathrm{m}^{-2}\right)\end{array}$ \\
\hline BIMA+JCMT & 2002 Apr 1 & $-4.3 \pm 0.1$ \\
$\ldots$ & 2004 Apr 1 & $-4.4 \pm 0.3$ \\
SMA & 2005 Jun 4 & $-6.7 \pm 2.9$ \\
$\ldots$ & 2005 Jun 6 & $-23.1 \pm 12.6$ \\
$\ldots$ & 2005 Jun 9 & $-5.0 \pm 1.7$ \\
$\ldots$ & 2005 Jun 15 & $-11.7 \pm 13.6$ \\
$\ldots$ & 2005 Jun 16 & $-5.4 \pm 1.8$ \\
$\ldots$ & 2005 Jun 17 & $-22.3 \pm 7.4$ \\
$\ldots$ & 2005 Jul 20 & $-7.5 \pm-.6$ \\
$\ldots$ & 2005 Jul 21 & $1.1 \pm 8.2$ \\
$\ldots$ & 2005 Jul 22 & $-3.7 \pm 1.8$ \\
$\ldots$ & 2005 Jul 30 & $-4.8 \pm 1.4$ \\
$\ldots$ & 2006 Jul 17 & $-5.6 \pm 1.6$ \\
\hline
\end{tabular}

small number of instances from the ensemble. We would, for instance, not clearly detect a red-noise spectrum with a characteristic timescale of $\gtrsim 10 \mathrm{yr}$ in this data. Nevertheless, we are able to draw some conclusions from this analysis. We observe more than an order of magnitude range in the SF on hour timescales. This range is somewhat reduced but still large when we excluded the largest RMs in epoch 1 . The dominant result from this structure function analysis is that we do not identify any characteristic timescale between hours and decades for variability of the RM. That is, RM variability is driven on a wide range of scales, from as close in as $10 R_{S}$ all the way out to the Bondi radius.

Pang et al. (2011) perform numerical simulations of magnetized accretion flows with weak convection to determine the timescale and magnitude of RM variations. Variability saturates on a Bondi time, $t_{B} \approx 100 \mathrm{yr}$, but can be significant on shorter timescales. The characteristic timescale for variability is determined as

$$
\tau=20\left(R_{\text {rel }} / R_{B}\right)^{2}\left(R_{\text {in }} / R_{B}\right)^{-1 / 2} t_{B}
$$

where $R_{\text {rel }} \sim 10 R_{S}$ is the radius at which electrons become relativistic, $R_{B}$ is the Bondi radius, and $R_{i n}$ is the reconnection scale in the simulation. For $R_{\text {in }} / R_{B}=10^{-5}$ as recommended by Pang et al. (2011), we calculate $\tau \sim 1 \mathrm{yr}$, with order-ofmagnitude uncertainty. It is difficult to reconcile the flat structure function that we observe with a distribution that has a characteristic timescale. The results suggest a more complex accretion flow structure than currently modeled or the existence of multiple processes contributing to RM variability.

Sharma et al. (2007) also performed MHD simulations and study of accretion flows with emphasis on geometry. Their conclusions focus on orientation effects. The equatorial plane of the accretion disk is modeled to be highly turbulent. Thus, a viewing angle through the plane would lead to RM sign reversals, which are not seen in our data. Note that the GC pulsar $\mathrm{RM}=-7 \times 10^{4} \mathrm{rad} \mathrm{m}^{-2}$ sets a threshold for the external RM that needs to be removed, but there are still no reversals that would be seen. On the other hand, polar viewing angles will produce a significant variability on a timescale of hours but with a consistent sign over long periods of time.

These ALMA observations were originally obtained with the goal of searching for RM changes due to disruption of the G2 cloud (Gillessen et al. 2012). Numerical models suggested that tidal forces could lead to a change in the accretion rate that would change the RM (e.g., Mościbrodzka et al. 2012). The cloud reached pericenter in 2014, approximately 2 yr before these observations were obtained. The cloud appeared to remain intact after close passage, although there may be largescale diffuse features (Plewa et al. 2017; Witzel et al. 2017). There is no evidence for enhanced accretion onto Sgr $\mathrm{A}^{*}$ (Haggard et al. 2014; Bower et al. 2015b). The epoch-averaged RM changes are linear with time, potentially the result of a secular change in RM but also within the bounds of archival variability and intra-epoch variability. Longer-term monitoring could detect a continuation of any secular trend and test the hypothesis of an enhanced accretion rate as the result of tidal streamers from $\mathrm{G} 2$ and other features.

\subsection{Polarization and Rotation Measure Variations within Epochs}

We see clear variations in the polarization properties within epochs, as well as between epochs. Total intensity variations within epochs are relatively small $(\sim 20 \%)$, and there are no well-defined flaring events in total intensity. The spectral index of the total intensity remains essentially flat during each epoch as well. Stokes $Q, U$, and $V$ flux densities, however, are seen to vary by as much as $100 \%$.

We show in Figure 12 that variations in the RM appear to be coupled with the LP flux density. The lower the polarization flux density, the higher the absolute value of the RM. The relation appears to have an inflection point near a polarized flux density of $100 \mathrm{mJy}$. Below this point, we see the $|\mathrm{RM}|$ become substantially larger than $10^{6} \mathrm{rad} \mathrm{m}^{-2}$. At high values of the polarization flux density, the RM asymptotes to a value near $-5 \times 10^{5} \mathrm{rad} \mathrm{m}^{-2}$. The large variations in $\mathrm{RM}$ do not contribute significantly to the epoch average because they are weighted by the polarization fraction. We find no correlation between variations in the $\mathrm{CP}$ fraction and the RM, as might be expected for the case where the $\mathrm{CP}$ is generated through conversion.

\subsection{Non-Faraday Variations}

We have focused on a Faraday interpretation for variations in the polarization angle with frequency and time. But it is also possible that intrinsic emission processes can produce similar 


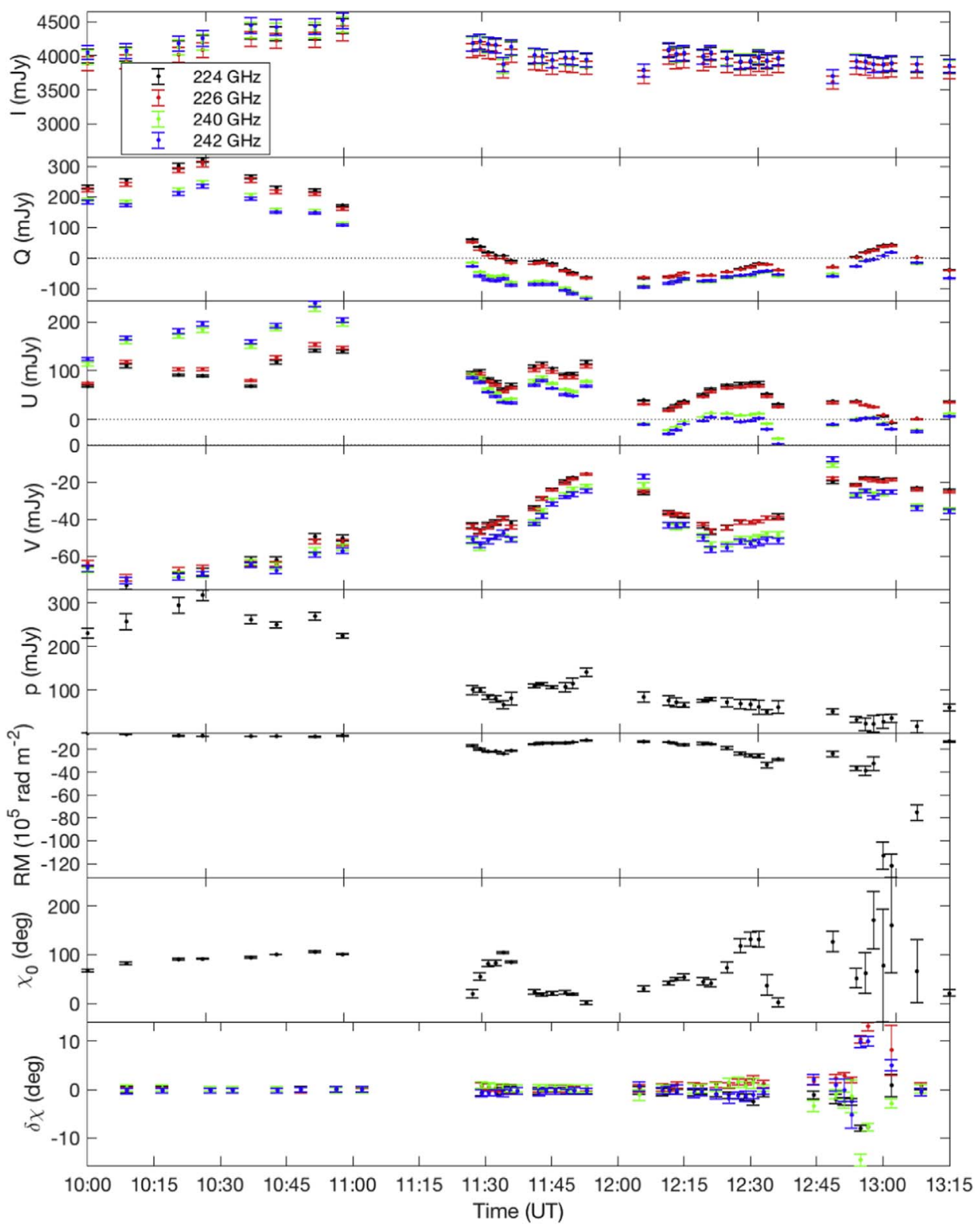

Figure 3. Timeseriess of Sgr $\mathrm{A}^{*}$ polarimetric data in epoch 1 . The top four panels show Stokes $I, Q, U$, and $V$ for each SPW. The fifth panel shows total linearly polarized intensity $(p)$. The sixth panel shows RM in units of $10^{5} \mathrm{rad} \mathrm{m}^{-2}$. The seventh panel shows the zero-wavelength position angle $\left(\chi_{0}\right)$. The eighth panel shows residual position angle after fitting the RM and position angle. Here multiple SPWs are shown: $224 \mathrm{GHz}$ (black), $226 \mathrm{GHz}$ (red), $240 \mathrm{GHz}$ (green), and $242 \mathrm{GHz}$ (blue).

effects. In particular, we know from millimeter VLBI polarimetry that the polarization structure of $\mathrm{Sgr} \mathrm{A}^{*}$ is not simple, i.e., not produced in a homogeneous structure with a single polarization structure (Johnson et al. 2015). While the VLBI observations cannot be uniquely translated into a map, they clearly require structure in the polarization vector field on scales smaller than the source size. Further, the fact that the total intensity spectrum peaks at millimeter wavelengths implies that some or all of the emission regions are near the optical depth unity surface (Bower et al. 2015b). Changes in individual regions in optical depth or polarization vector magnitude or orientation can lead to changes in the integrated polarization as a function of wavelength. This could lead to destructive interference of the polarized signature, a change in the apparent RM, or non- $\lambda^{2}$ effects.
One of the best tests of intrinsic rather than Faraday origins for polarization variability is to search for deviations from the $\lambda^{2}$ law. In Figures 3-5, we show position angle residuals after fitting an RM to each integration for Sgr A*. For epochs 2 and 3, the data are all consistent with a Faraday interpretation. But for the end of epoch 1, we see large residuals, suggesting that a Faraday interpretation is not a good fit. The largest deviations from a Faraday interpretation occur for apparent $\mathrm{RM}>\mathrm{few} \times$ $10^{6} \mathrm{rad} \mathrm{m}^{-2}$, or polarized intensity $<100 \mathrm{mJy}$. These differences are also apparent in Figures 6-8.

The interpretation of these complex wavelength-dependent polarization effects will likely require comparison with numerical modeling efforts (Shcherbakov et al. 2012; Gold et al. 2017; Jiménez-Rosales \& Dexter 2018; Mościbrodzka \& Gammie 2018). 


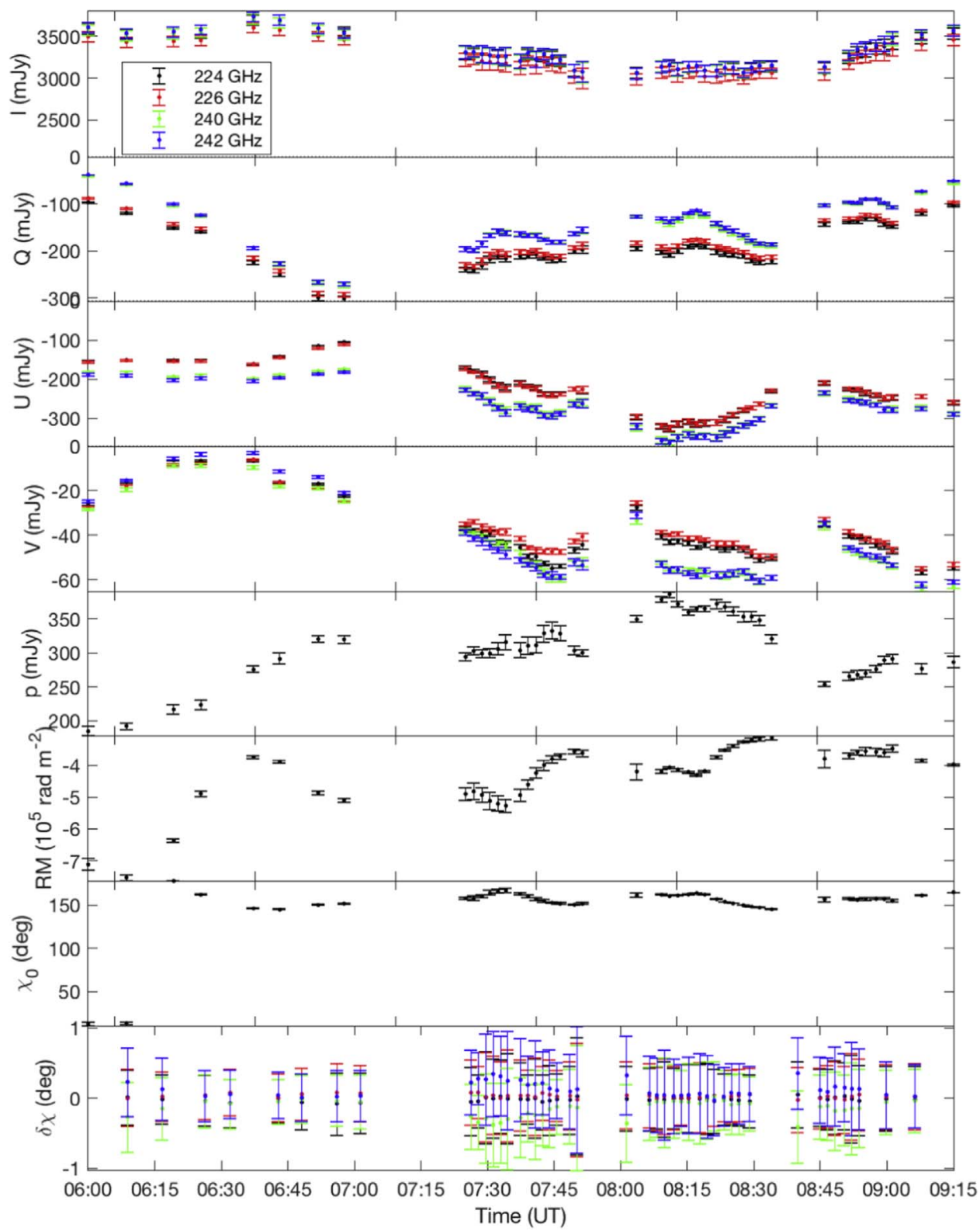

Figure 4. Same as Figure 3, but for epoch 2.

\section{Conclusions}

These new ALMA results suggest a physical model of longterm stability coupled with short-term variability in the polarization properties. The long-term stability in the Faraday rotation suggests a stable magnetic field configuration and an origin for much of the Faraday rotation at large radii from Sgr A*. These support the hypothesis that the average RM is a useful constraint on the mean accretion flow properties. The variations in the RM on timescales of months are a potentially useful diagnostic of the scale of turbulent or secular fluctuations in the accretion flow properties. Although we did not see any effects clearly related to passing of the G2 cloud, the sensitivity of these measurements confirms that future interactions may produce detectable Faraday signatures.

The consistency of the sign of $\mathrm{CP}$ over a wide range of wavelengths and decades in time suggests a stable magnetic field configuration on scales from a few $R_{S}$ to hundreds of $R_{S}$.
This stability is probably best achieved if the $\mathrm{CP}$ is arising through Faraday conversion in emission regions with poloidal magnetic fields. In this model, we exclude an edge-on geometry. Alternatively, Doppler boosting in a toroidal magnetic field configuration could lead to a persistent asymmetry that produces a consistent sign.

The short-term variations observed suggest a complex scenario on scales of a few to $\sim 10 R_{S}$, in which both emission and propagation effects are important. LP and CP are variable on timescales of hours, comparable to the Keplerian timescale at these small radii. The apparent relationship between changes in LP and wavelength-dependent effects suggests that the mildly relativistic electrons that are responsible for the synchrotron emission also contribute to propagation effects. It is unclear whether variations that are modeled as the RM are truly propagation effects or are the result of a complex, partially optically thick surface from which the emission originates. This 


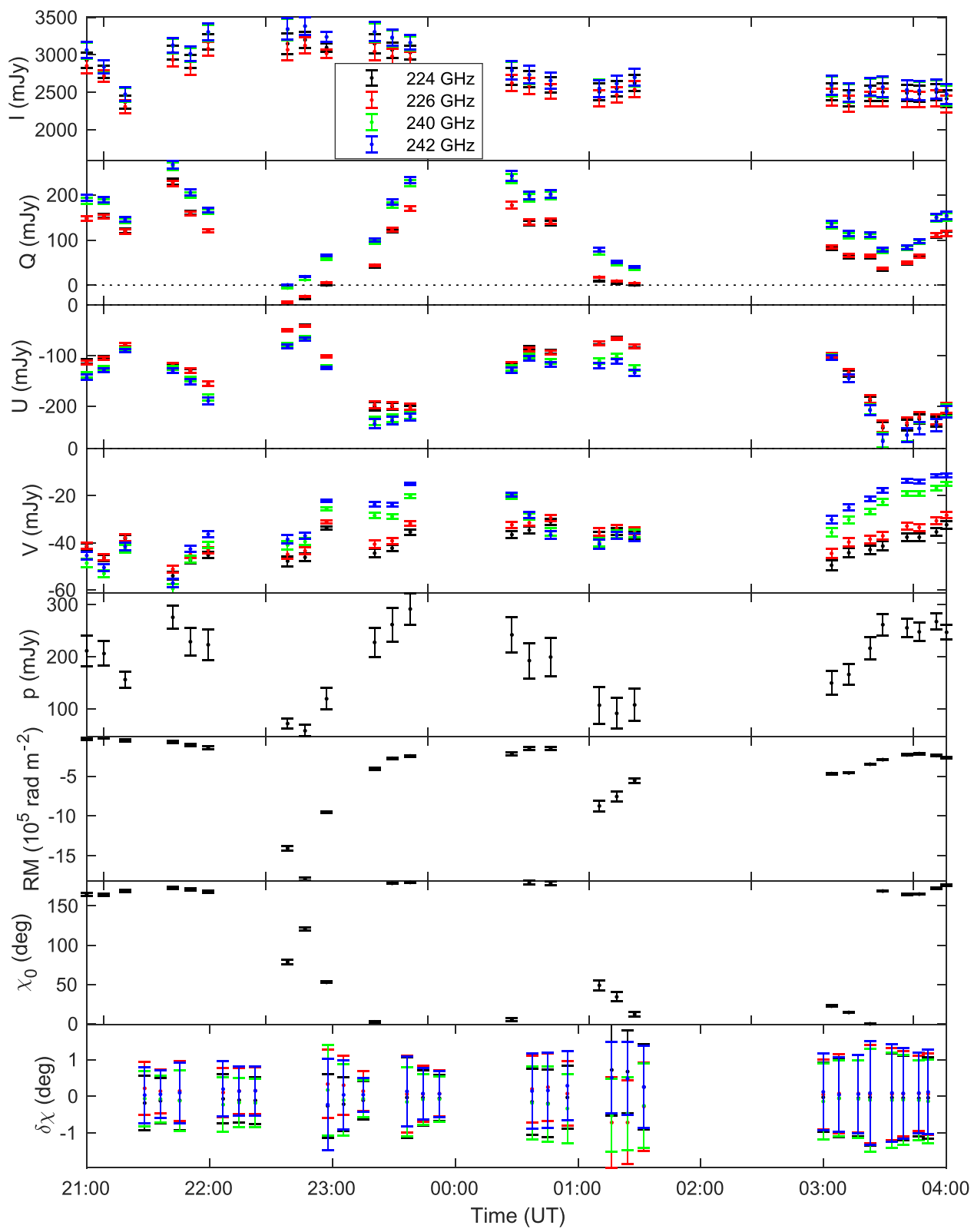

Figure 5. Same as Figure 3, but for epoch 3.

picture is consistent with EHT polarimetric models of Sgr A* that reveal polarimetric structure on scales smaller than the total intensity region.

We have been conservative in our analysis of systematic errors based on calibrator observations and do not claim detection of Faraday rotation toward these sources. But it is possible that these calibrators do in fact reveal large RMs that are indicative of dense, magnetized plasma in the inner regions of these sources.

Separating intrinsic and propagation effects in Sgr A* can be achieved through several approaches. Longer-term monitoring of Sgr $\mathrm{A}^{*}$ with intervals of days to years can provide a more accurate and complete picture of the scale on which variations originate. Our data are undersampled for establishing the nature of variations on timescales shorter than years. Simultaneous measurements at a wider range of wavelengths, especially at wavelengths longer than $1.3 \mathrm{~mm}$ where Faraday effects are stronger, will be an important probe of non- $\lambda^{2}$ effects that are predicted for models of mixed emission and propagation effects. Shorter-wavelength observations may provide more direct probes of the intrinsic emission process as Faraday effects weaken and the emission region shrinks.

The EHT will obtain polarimetric images of Sgr A* with sensitivity and fidelity that is substantially improved over past results. We expect that images could reveal polarimetric structures with independent intrinsic and Faraday characteristics. Localized $\mathrm{CP}$ signatures can give insights into the conversion mechanism. Analysis of EHT data must be carried out in a domain that is time dependent, frequency-dependent, and adaptable to complex Faraday mechanisms.

This paper makes use of the following ALMA data: ADS/ JAO.ALMA\#2013.1.00764.S. ALMA is a partnership of ESO (representing its member states), NSF (USA), and NINS 


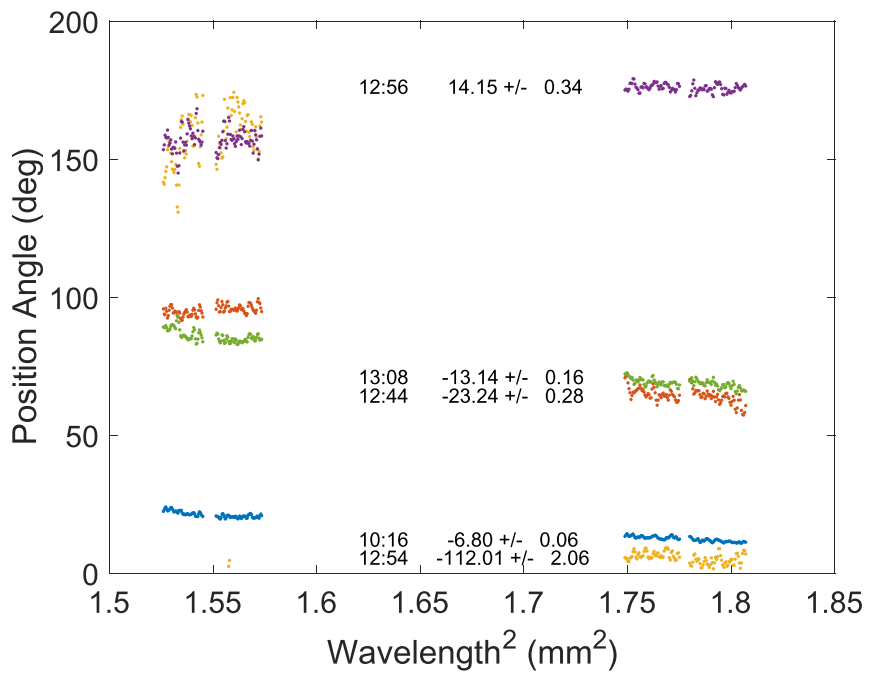

Figure 6. Polarization angle vs. wavelength squared for a selected set of scans from epoch 1. The labels give the time of the scan and the fitted RM in units of $10^{5} \mathrm{rad} \mathrm{m}^{-2}$ and are aligned with the longer-wavelength points to their right.

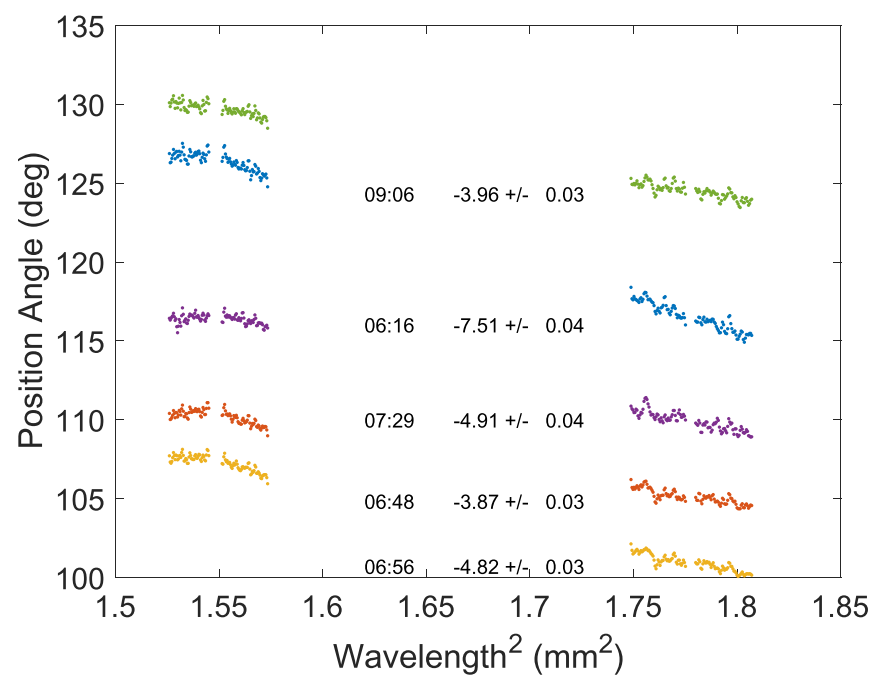

Figure 7. Polarization angle vs. wavelength squared for a selected set of scans from epoch 2. The labels give the time of the scan and the fitted RM in units of $10^{5} \mathrm{rad} \mathrm{m}^{-2}$ and are aligned with the longer-wavelength points to their right.

(Japan), together with NRC (Canada), MOST and ASIAA (Taiwan), and KASI (Republic of Korea), in cooperation with the Republic of Chile. The Joint ALMA Observatory is operated by ESO, AUI/NRAO, and NAOJ. The National Radio Astronomy Observatory is a facility of the National Science Foundation operated under cooperative agreement by Associated Universities, Inc. H.F. acknowledges funding from the European Research Council (ERC) Synergy Grant "BlackHoleCam" (grant 610058).

Facility: ALMA.

Software: CASA, MATLAB.

\section{Appendix A}

\section{Calibrator Results and Estimates of Systematic Errors}

We use calibrator results in this section to set limits on systematic errors on LP fraction, CP fraction, and RM. We set these limits for inter-epoch and intra-epoch comparisons and find that they are comparable (Table 5). Our CASA analysis

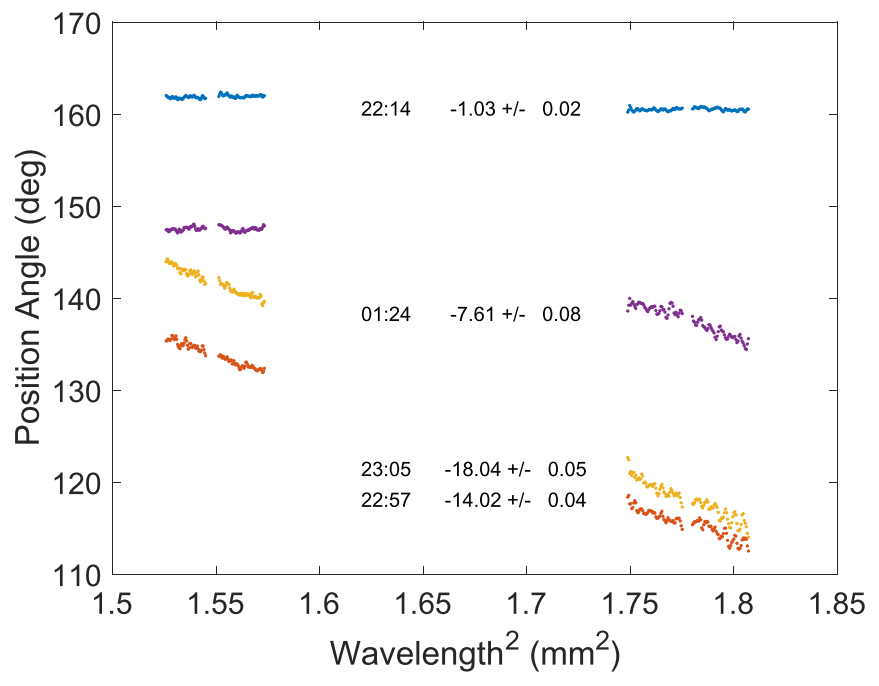

Figure 8. Polarization angle vs. wavelength squared for a selected set of scans from epoch 3 . The labels give the time of the scan and the fitted RM in units of $10^{5} \mathrm{rad} \mathrm{m}^{-2}$ and are aligned with the longer-wavelength points to their right.

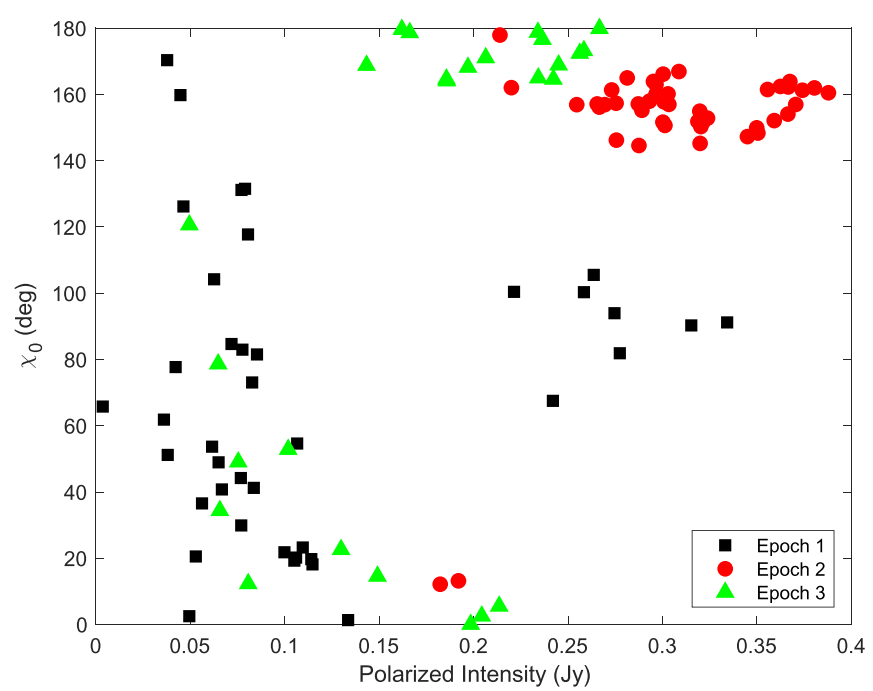

Figure 9. Zero-wavelength position angle as a function of polarized intensity for Sgr $\mathrm{A}^{*}$ in three epochs.

was similar to that employed for science verification data, which found a characteristic error $\delta \chi=0.4$ and errors in fractional LP $<0.1 \%$ (Nagai et al. 2016).

We present similar analysis results for the calibrators as for Sgr A* to facilitate comparison and demonstration of the reliability of our main results. Figure 13 shows the SPW-averaged polarization angles and fitted RMs for all calibrators. Figure 14 shows the channel-averaged polarization angles for each epoch as well. Time-dependent Stokes parameters, fitted RMs, position angles, and residuals are shown in Figures 15-23. In Table 6 we present the epoch-averaged polarization properties as a function of SPW. In Table 7, we present derived polarization properties for the calibrators in each epoch, including RM.

Treating these calibrator results as limits of calibration accuracy must be given with the caveat that there is evidence of large RMs toward some AGNs at millimeter wavelengths. In particular, 3C 84 shows an $\mathrm{RM} \approx 10^{6} \mathrm{rad} \mathrm{m}^{-2}$ (Plambeck et al. 2014), and M87 shows an $\mathrm{RM} \approx 10^{5} \mathrm{rad} \mathrm{m}^{-2}$ (Kuo et al. 2014). ALMA observations of 3C 273 reveal 


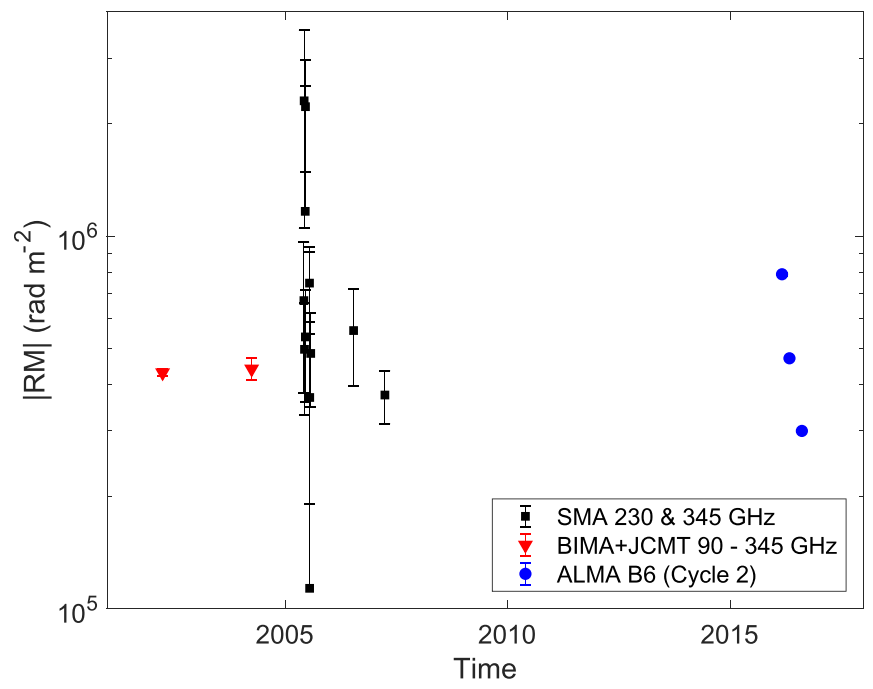

Figure 10. RM absolute value vs. time from these new and previously published measurements. BIMA+JCMT measurements (Bower et al. 2003 Macquart et al. 2006) were widely separated in time but covered a broad frequency range; thus, they have a small statistical error but a large systematic uncertainty due to variability. SMA measurements were obtained simultaneously but with a small frequency range (Marrone et al. 2007). ALMA measurements are averaged over multihour epochs, which show significant variability in the RM on shorter timescales. ALMA error bars are smaller than the displayed points.

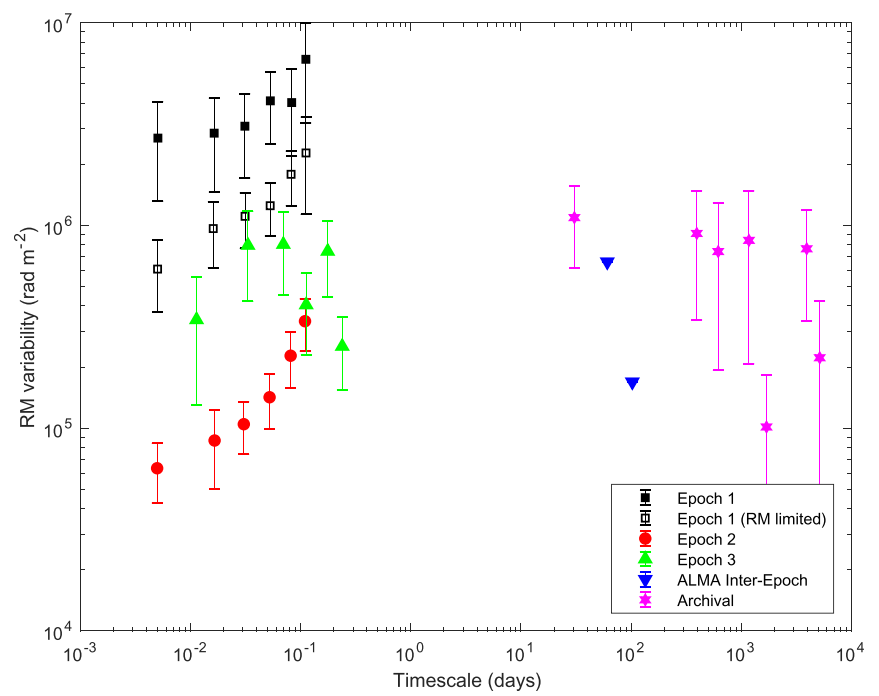

Figure 11. RM variability as a function of timescale. We show the square root of the structure function calculated for individual epochs and in between epochs. Open symbols for epoch 1 are calculated excluding the largest and most ambiguous RMs with $|\mathrm{RM}|>5 \times 10^{6} \mathrm{rad} \mathrm{m}^{-2}$.

$\mathrm{RM}=(3.6 \pm 0.3) \times 10^{5} \mathrm{rad} \mathrm{m}^{-2} \quad$ (Hovatta et al. 2018). Trippe et al. (2012) report RMs as large as $10^{5} \mathrm{rad} \mathrm{m}^{-2}$ for AGNs at wavelengths near millimeter wavelength. These large RMs are interpreted as originating from the accretion flow, the relativistic jet, or the dense gas of the nuclear region (e.g., Mościbrodzka et al. 2017). Therefore, there is possibly a significant intrinsic contribution to these observed RMs, although it cannot be quantified for these sources from these data.

\section{A.1. Inter-epoch Properties}

The most optimistic limits on polarization calibration are set by analysis of the polarization calibrator, J1751+0939. The
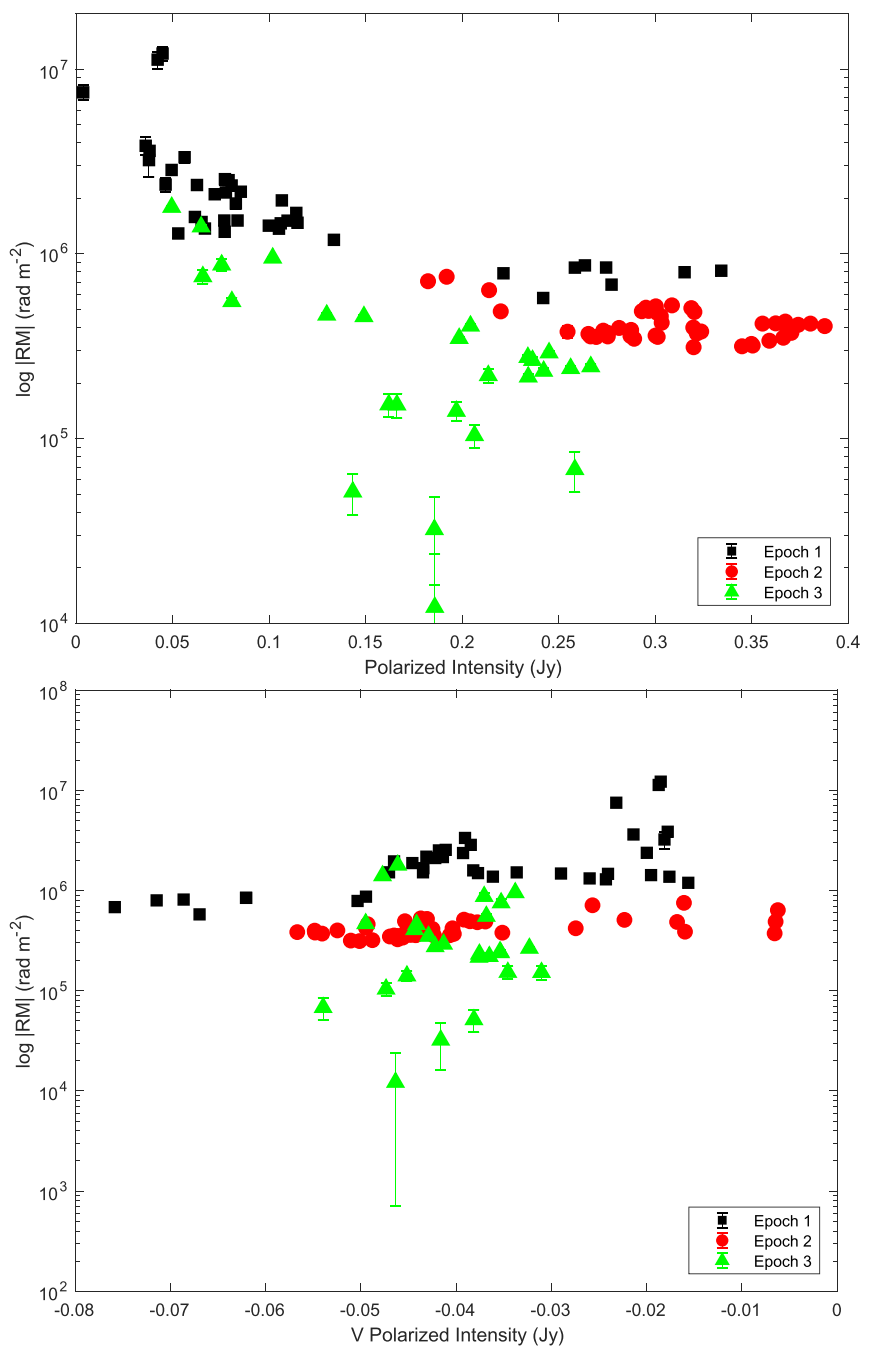

Figure 12. Linearly (top) and circularly (bottom) polarized intensity vs. $|\mathrm{RM}|$ for $\operatorname{Sgr} \mathrm{A}^{*}$ in three epochs.

Table 5

Systematic Limits on Polarization Properties

\begin{tabular}{lcc}
\hline \hline LP Fraction & CP Fraction & RM \\
\hline $0.1 \%$ & $0.2 \%$ & $10^{5} \mathrm{rad} \mathrm{m}^{-2}$ \\
\hline
\end{tabular}

polarization calibration assumes $V=0$, which is achieved with an accuracy of $\lesssim 0.1 \%$ for the calibrator, J1751+039. The mean polarization angle for J1751+0939 within an epoch is determined to an accuracy of $<0^{\circ}$. 1 . The RM for each epoch is constrained to less than a few $\times 10^{4} \mathrm{rad} \mathrm{m}^{-2}$.

The results obtained for the other calibrators provide a more realistic estimate of accuracy of polarization calibration and gain solution transfer. In particular, the phase calibrator, J1733-3722, and the check source, J1713-3418, are observed with similar cycles to Sgr A $^{*}$. J1713-3418 is consistent with a mean $\mathrm{RM}=-0.68 \times 10^{5} \pm 0.59 \times 10^{5} \mathrm{rad} \mathrm{m}^{-2} . \mathrm{J} 1733-3722$ is inconsistent with a constant RM. Consistency can be forced by adding in quadrature an error of $0.6 \times 10^{5} \mathrm{rad} \mathrm{m}^{-2}$. The upper bound on RM found in any epoch for these sources is epoch 1 for J1733-3722 with a value of 

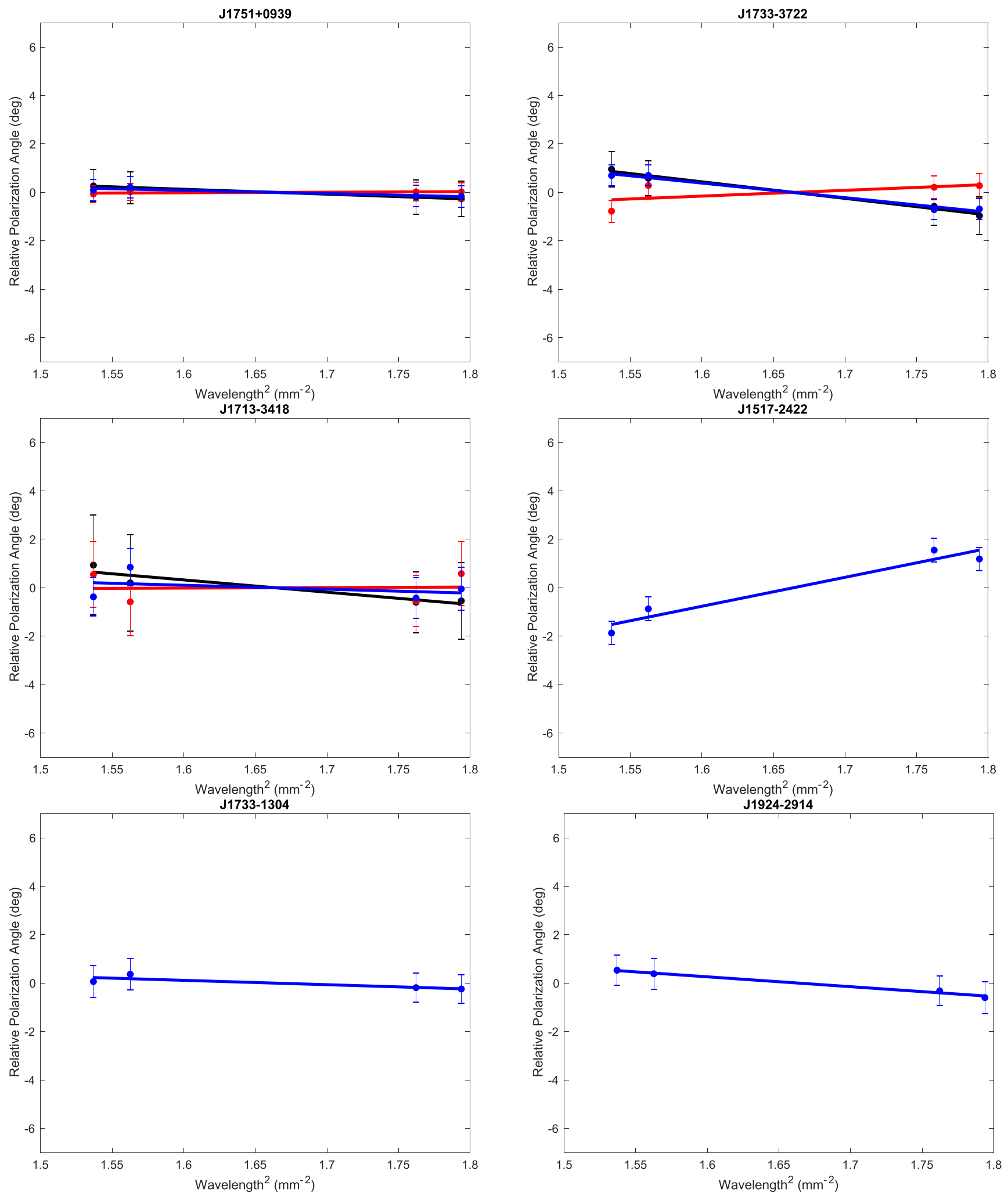

Figure 13. Average residual polarization position angle as a function of wavelength squared for each of the calibrators in epochs 1 (black), 2 (red), and 3 (blue). We have removed the mean position angle for each source in each epoch to enable clear comparison. All plots are on the same scale for wavelength-scaled and position angle.

$-1.19 \times 10^{5} \pm 0.10 \times 10^{5} \mathrm{rad} \mathrm{m}^{-2}$. We find similar limits for J1733-1304 and J1924-2914 but a somewhat large value for a one-epoch limit for J1517-2422 $\left(2.09 \times 10^{5} \pm\right.$ $0.38 \times 10^{5} \mathrm{rad} \mathrm{m}^{-2}$ ). The limits for these sources are less reliable because they are the result of only single snapshot observations, whereas J1733-3722 and J1713-3418 were observed $>10$ and $>5$ times per epoch, respectively, over a wide range of parallactic angles. 

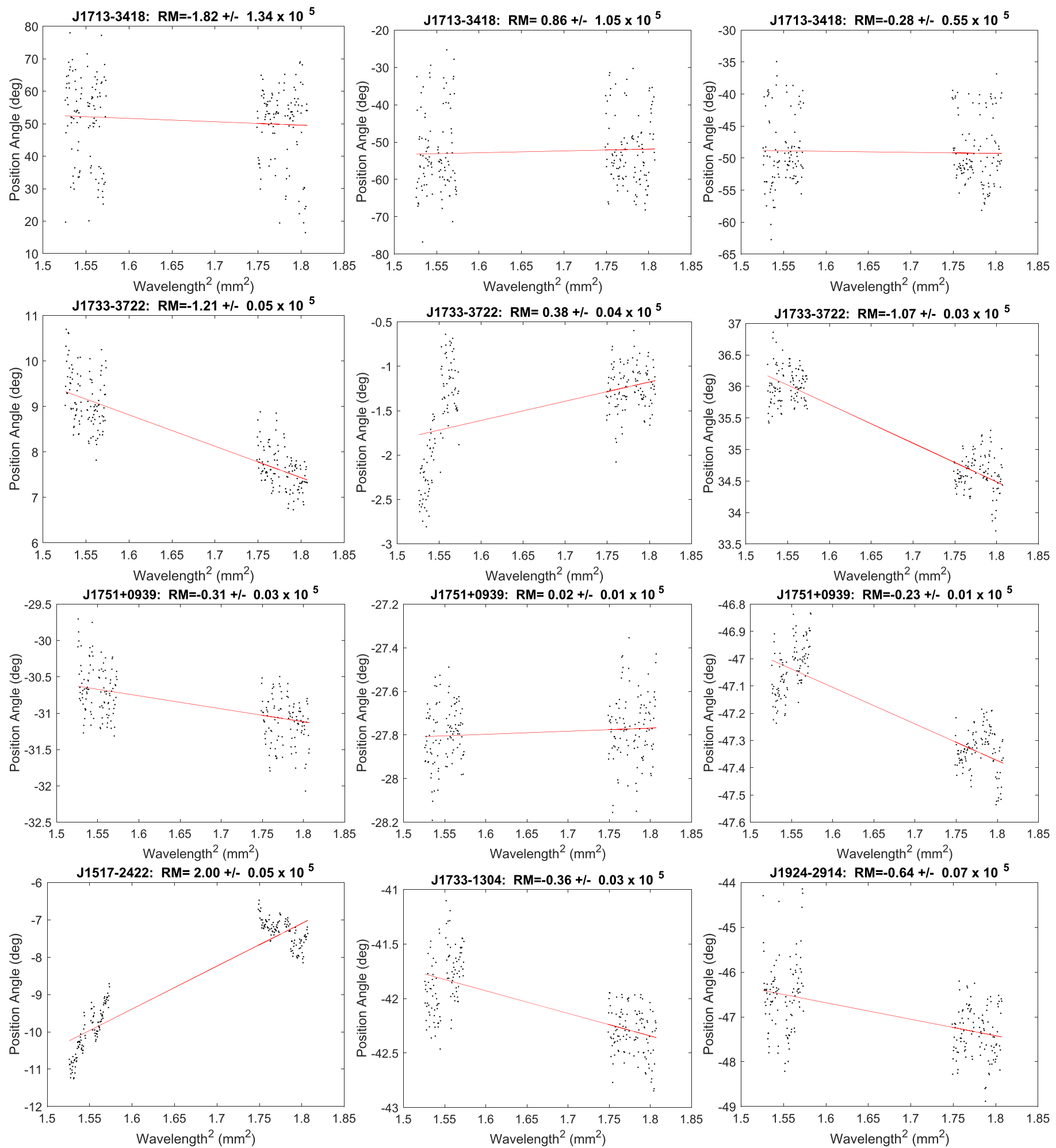

Figure 14. Polarization angle as a function of wavelength squared, presented for each channel and for each epoch for all calibrators. Epochs 1, 2, and 3 are in left, middle, and right columns, respectively, except for the last row. In the last row, the three calibrators included only in epoch 3 are shown. Note the different scales for position angle. The title gives the RM units of $\mathrm{rad} \mathrm{m}^{-2}$.

We use the calibrators and estimates of intrinsic RM to establish a systematic limit of $10^{5} \mathrm{rad} \mathrm{m}^{-2}$ for changes between sources and epochs. This limit corresponds to a change in the position angle across the band of 1.3 . As discussed below, we estimate that systematic errors within an epoch for a given source are less than this value.
Calibration of Stokes $V$ for polarization and gain assumes $V=0$. As a result, we can only set systematic limits on Stokes $V$ with the check source J1713-3418. The gain calibration for J1713-3418 is shared with that of Sgr A*. Stokes V is detected for this check source with statistical significance in only epoch 2 at a level $V \lesssim 0.2 \%$, 


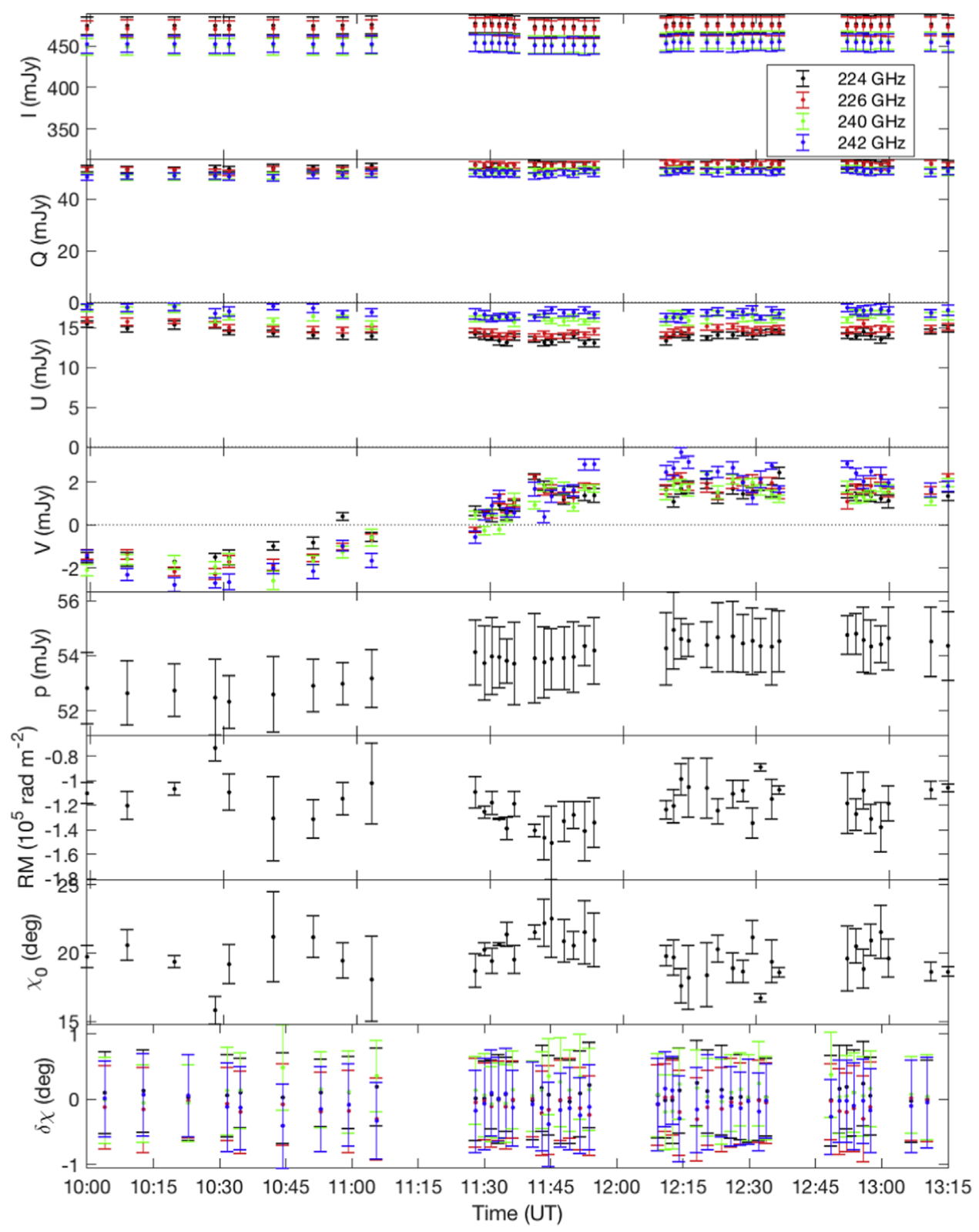

Figure 15. Timeseries plot for J1733-3722 in epoch 1 following Figure 3.

which we adopt as our systematic threshold for detection and change between sources and epochs. We note that in its proposal materials for Cycle 6 ALMA suggests a
CP systematic error of $0.6 \%$. Detailed analysis for ALMA observations of 3C 273 finds $V=0.2 \%$ (Hovatta et al. 2018). 
Table 6

Calibrator Average Polarization Properties

\begin{tabular}{|c|c|c|c|c|c|c|}
\hline Source & Epoch & SPW & $\begin{array}{c}I \\
(\mathrm{mJy})\end{array}$ & $\underset{(\mathrm{mJy})}{Q}$ & $\begin{array}{c}U \\
(\mathrm{mJy})\end{array}$ & $\begin{array}{c}V \\
(\mathrm{mJy})\end{array}$ \\
\hline J1713-3418 & 1 & 0 & $234 \pm 7$ & $-0.763 \pm 0.157$ & $3.069 \pm 0.175$ & $-0.040 \pm 0.133$ \\
\hline$\ldots$ & $\ldots$ & 1 & $232 \pm 7$ & $-0.762 \pm 0.157$ & $3.091 \pm 0.138$ & $0.030 \pm 0.140$ \\
\hline$\ldots$ & $\ldots$ & 2 & $222 \pm 6$ & $-0.753 \pm 0.173$ & $2.725 \pm 0.198$ & $0.373 \pm 0.159$ \\
\hline$\ldots$ & $\ldots$ & 3 & $224 \pm 6$ & $-0.913 \pm 0.235$ & $3.002 \pm 0.225$ & $-0.412 \pm 0.220$ \\
\hline J1713-3418 & 2 & 0 & $243 \pm 4$ & $-0.906 \pm 0.246$ & $-3.697 \pm 0.169$ & $0.251 \pm 0.152$ \\
\hline$\ldots$ & $\ldots$ & 1 & $242 \pm 4$ & $-1.208 \pm 0.152$ & $-4.203 \pm 0.161$ & $0.377 \pm 0.172$ \\
\hline$\ldots$ & $\ldots$ & 2 & $230 \pm 4$ & $-1.120 \pm 0.172$ & $-3.881 \pm 0.201$ & $0.426 \pm 0.134$ \\
\hline$\ldots$ & $\ldots$ & 3 & $230 \pm 4$ & $-1.169 \pm 0.219$ & $-4.744 \pm 0.232$ & $0.620 \pm 0.210$ \\
\hline J1713-3418 & 3 & 0 & $196 \pm 4$ & $-0.667 \pm 0.133$ & $-3.920 \pm 0.123$ & $-0.336 \pm 0.112$ \\
\hline$\ldots$ & $\ldots$ & 1 & $195 \pm 4$ & $-0.691 \pm 0.077$ & $-3.764 \pm 0.112$ & $-0.213 \pm 0.084$ \\
\hline$\ldots$ & $\ldots$ & 2 & $193 \pm 4$ & $-0.576 \pm 0.091$ & $-4.173 \pm 0.112$ & $0.206 \pm 0.081$ \\
\hline$\ldots$ & $\ldots$ & 3 & $193 \pm 4$ & $-0.753 \pm 0.099$ & $-4.140 \pm 0.117$ & $0.242 \pm 0.102$ \\
\hline J1733-3722 & 1 & 0 & $475 \pm 14$ & $53.035 \pm 1.535$ & $14.043 \pm 0.397$ & $0.817 \pm 0.061$ \\
\hline$\ldots$ & $\ldots$ & 1 & $472 \pm 14$ & $52.687 \pm 1.524$ & $14.717 \pm 0.422$ & $0.882 \pm 0.064$ \\
\hline$\ldots$ & $\ldots$ & 2 & $454 \pm 12$ & $50.531 \pm 1.382$ & $16.346 \pm 0.451$ & $0.739 \pm 0.064$ \\
\hline$\ldots$ & $\ldots$ & 3 & $453 \pm 12$ & $50.036 \pm 1.398$ & $16.908 \pm 0.467$ & $0.999 \pm 0.060$ \\
\hline J1733-3722 & 2 & 0 & $648 \pm 11$ & $34.860 \pm 0.601$ & $-1.448 \pm 0.060$ & $0.191 \pm 0.048$ \\
\hline$\cdots$ & $\cdots$ & 1 & $645 \pm 11$ & $34.557 \pm 0.573$ & $-1.515 \pm 0.042$ & $0.146 \pm 0.065$ \\
\hline$\ldots$ & $\ldots$ & 2 & $620 \pm 10$ & $32.813 \pm 0.506$ & $-1.354 \pm 0.055$ & $-0.159 \pm 0.051$ \\
\hline$\ldots$ & $\ldots$ & 3 & $618 \pm 10$ & $32.421 \pm 0.521$ & $-2.542 \pm 0.062$ & $-0.355 \pm 0.056$ \\
\hline J1733-3722 & 3 & 0 & $570 \pm 9$ & $11.000 \pm 0.194$ & $29.049 \pm 0.487$ & $0.266 \pm 0.105$ \\
\hline$\ldots$ & $\ldots$ & 1 & $569 \pm 9$ & $11.050 \pm 0.194$ & $29.101 \pm 0.475$ & $-0.226 \pm 0.048$ \\
\hline$\ldots$ & $\ldots$ & 2 & $565 \pm 9$ & $9.529 \pm 0.169$ & $29.396 \pm 0.482$ & $-0.441 \pm 0.052$ \\
\hline$\ldots$ & $\ldots$ & 3 & $563 \pm 9$ & $9.507 \pm 0.169$ & $29.304 \pm 0.483$ & $-0.448 \pm 0.068$ \\
\hline $\mathrm{J} 1751+0939$ & 1 & 0 & $1905 \pm 59$ & $14.873 \pm 0.459$ & $-28.325 \pm 0.882$ & $-0.097 \pm 0.068$ \\
\hline$\ldots$ & $\ldots$ & 1 & $1896 \pm 58$ & $14.936 \pm 0.436$ & $-28.282 \pm 0.870$ & $-0.015 \pm 0.067$ \\
\hline$\ldots$ & $\ldots$ & 2 & $1820 \pm 52$ & $14.795 \pm 0.416$ & $-27.112 \pm 0.778$ & $-0.033 \pm 0.083$ \\
\hline$\ldots$ & $\ldots$ & 3 & $1846 \pm 53$ & $15.082 \pm 0.415$ & $-27.476 \pm 0.808$ & $-0.196 \pm 0.073$ \\
\hline $\mathrm{J} 1751+0939$ & 2 & 0 & $1964 \pm 35$ & $33.880 \pm 0.577$ & $-49.329 \pm 0.865$ & $-0.244 \pm 0.059$ \\
\hline$\ldots$ & $\ldots$ & 1 & $1954 \pm 34$ & $33.720 \pm 0.598$ & $-49.098 \pm 0.874$ & $-0.208 \pm 0.051$ \\
\hline$\ldots$ & $\ldots$ & 2 & $1876 \pm 31$ & $32.353 \pm 0.525$ & $-47.128 \pm 0.776$ & $-0.290 \pm 0.083$ \\
\hline$\ldots$ & $\ldots$ & 3 & $1886 \pm 31$ & $32.294 \pm 0.533$ & $-47.337 \pm 0.765$ & $-0.352 \pm 0.074$ \\
\hline $\mathrm{J} 1751+0939$ & 3 & 0 & $2342 \pm 36$ & $-7.714 \pm 0.139$ & $-93.228 \pm 1.435$ & $-0.197 \pm 0.106$ \\
\hline$\ldots$ & $\ldots$ & 1 & $2340 \pm 36$ & $-7.668 \pm 0.113$ & $-93.420 \pm 1.426$ & $-0.074 \pm 0.050$ \\
\hline$\cdots$ & $\cdots$ & 2 & $2327 \pm 36$ & $-6.473 \pm 0.113$ & $-93.514 \pm 1.454$ & $0.204 \pm 0.055$ \\
\hline$\ldots$ & $\cdots$ & 3 & $2325 \pm 36$ & $-6.883 \pm 0.118$ & $-93.509 \pm 1.457$ & $0.142 \pm 0.058$ \\
\hline $\mathrm{J} 1517-2422$ & 3 & 0 & $2963 \pm 53$ & $69.603 \pm 1.255$ & $-18.583 \pm 0.367$ & $-0.185 \pm 0.171$ \\
\hline$\ldots$ & $\ldots$ & 1 & $2971 \pm 53$ & $70.258 \pm 1.274$ & $-17.801 \pm 0.334$ & $0.275 \pm 0.106$ \\
\hline$\ldots$ & $\ldots$ & 2 & $2966 \pm 56$ & $77.661 \pm 1.468$ & $-26.849 \pm 0.516$ & $1.677 \pm 0.144$ \\
\hline$\ldots$ & $\ldots$ & 3 & $2968 \pm 56$ & $77.915 \pm 1.482$ & $-30.013 \pm 0.571$ & $2.605 \pm 0.180$ \\
\hline J1733-1304 & 3 & 0 & $2191 \pm 46$ & $10.036 \pm 0.283$ & $-106.769 \pm 2.242$ & $0.600 \pm 0.294$ \\
\hline$\ldots$ & $\cdots$ & 1 & $2188 \pm 46$ & $10.301 \pm 0.253$ & $-107.436 \pm 2.256$ & $0.273 \pm 0.158$ \\
\hline$\ldots$ & $\ldots$ & 2 & $2156 \pm 49$ & $12.350 \pm 0.323$ & $-106.954 \pm 2.437$ & $3.158 \pm 0.157$ \\
\hline$\ldots$ & $\ldots$ & 3 & $2150 \pm 49$ & $11.136 \pm 0.344$ & $-106.260 \pm 2.456$ & $3.572 \pm 0.178$ \\
\hline J1924-2914 & 3 & 0 & $3784 \pm 79$ & $-3.980 \pm 0.206$ & $-44.584 \pm 1.026$ & $-5.749 \pm 0.514$ \\
\hline$\ldots$ & $\ldots$ & 1 & $3769 \pm 80$ & $-3.408 \pm 0.169$ & $-42.889 \pm 0.914$ & $-3.519 \pm 0.429$ \\
\hline$\cdots$ & $\ldots$ & 2 & $3706 \pm 79$ & $-2.062 \pm 0.176$ & $-37.685 \pm 0.835$ & $-1.293 \pm 0.341$ \\
\hline$\ldots$ & $\ldots$ & 3 & $3686 \pm 79$ & $-1.899 \pm 0.217$ & $-38.272 \pm 0.832$ & $0.510 \pm 0.336$ \\
\hline
\end{tabular}


Table 7

Calibrator Frequency-averaged Polarization and Rotation Measures

\begin{tabular}{|c|c|c|c|c|c|c|c|c|c|}
\hline Source & Epoch & $\begin{array}{c}I \\
(\mathrm{mJy})\end{array}$ & $\begin{array}{c}\delta I \\
\left(\mathrm{mJy} \mathrm{GHz}^{-1}\right)\end{array}$ & $\begin{array}{c}P \\
(\mathrm{mJy})\end{array}$ & $\begin{array}{c}\delta P \\
\left(\mathrm{mJy} \mathrm{GHz}^{-1}\right)\end{array}$ & $\begin{array}{c}V \\
(\mathrm{mJy})\end{array}$ & $\begin{array}{c}\delta V \\
\left(\mathrm{mJy} \mathrm{GHz}^{-1}\right)\end{array}$ & $\begin{array}{c}\mathrm{RM} \\
\left(10^{5} \mathrm{rad} \mathrm{m}^{-2}\right)\end{array}$ & $\begin{array}{c}\chi_{0} \\
(\mathrm{deg})\end{array}$ \\
\hline J1713-3418 & 1 & $228 \pm 1$ & $-0.6 \pm 0.8$ & $3.07 \pm 0.07$ & $-0.01 \pm 0.01$ & $0.045 \pm 0.169$ & $0.005 \pm 0.021$ & $-0.88 \pm 0.24$ & $61.0 \pm 2.3$ \\
\hline$\ldots$ & 2 & $237 \pm 0$ & $-0.8 \pm 0.4$ & $4.27 \pm 0.23$ & $0.02 \pm 0.03$ & $0.397 \pm 0.046$ & $0.012 \pm 0.006$ & $0.04 \pm 0.61$ & $-52.8 \pm 5.8$ \\
\hline$\cdots$ & 3 & $194 \pm 0$ & $-0.2 \pm 0.1$ & $4.05 \pm 0.05$ & $0.02 \pm 0.01$ & $-0.020 \pm 0.016$ & $0.031 \pm 0.002$ & $-0.28 \pm 0.51$ & $-47.1 \pm 4.9$ \\
\hline J1733-3722 & 1 & $463 \pm 0$ & $-1.3 \pm 0.4$ & $53.87 \pm 0.02$ & $-0.11 \pm 0.00$ & $0.863 \pm 0.066$ & $0.003 \pm 0.008$ & $-1.19 \pm 0.10$ & $19.8 \pm 1.0$ \\
\hline$\ldots$ & 2 & $633 \pm 0$ & $-1.7 \pm 0.4$ & $33.71 \pm 0.02$ & $-0.13 \pm 0.00$ & $-0.041 \pm 0.036$ & $-0.027 \pm 0.004$ & $0.42 \pm 0.38$ & $-5.4 \pm 3.6$ \\
\hline$\cdots$ & 3 & $567 \pm 0$ & $-0.4 \pm 0.3$ & $30.98 \pm 0.03$ & $-0.01 \pm 0.00$ & $-0.288 \pm 0.084$ & $-0.021 \pm 0.011$ & $-1.05 \pm 0.10$ & $45.3 \pm 1.0$ \\
\hline $\mathrm{J} 1751+0939$ & 1 & $1866 \pm 9$ & $-4.1 \pm 9.2$ & $31.54 \pm 0.15$ & $-0.05 \pm 0.02$ & $-0.090 \pm 0.043$ & $-0.004 \pm 0.005$ & $-0.35 \pm 0.01$ & $-27.5 \pm 0.1$ \\
\hline$\cdots$ & 2 & $1920 \pm 5$ & $-4.8 \pm 5.1$ & $58.47 \pm 0.12$ & $-0.15 \pm 0.01$ & $-0.273 \pm 0.018$ & $-0.006 \pm 0.002$ & $0.04 \pm 0.03$ & $-28.2 \pm 0.3$ \\
\hline$\cdots$ & 3 & $2334 \pm 0$ & $-0.9 \pm 0.1$ & $93.70 \pm 0.04$ & $0.01 \pm 0.01$ & $0.034 \pm 0.031$ & $0.017 \pm 0.004$ & $-0.23 \pm 0.06$ & $-45.0 \pm 0.6$ \\
\hline $\mathrm{J} 1517-2422$ & 3 & $2967 \pm 2$ & $0.1 \pm 2.2$ & $77.55 \pm 0.24$ & $0.66 \pm 0.03$ & $1.084 \pm 0.158$ & $0.127 \pm 0.021$ & $2.09 \pm 0.38$ & $-28.6 \pm 3.7$ \\
\hline J1733-1304 & 3 & $2171 \pm 0$ & $-2.3 \pm 0.4$ & $107.42 \pm 0.27$ & $-0.02 \pm 0.03$ & $1.804 \pm 0.139$ & $0.194 \pm 0.018$ & $-0.31 \pm 0.13$ & $-39.1 \pm 1.3$ \\
\hline J1924-2914 & 3 & $3736 \pm 3$ & $-5.1 \pm 2.6$ & $40.93 \pm 0.42$ & $-0.36 \pm 0.05$ & $-2.462 \pm 0.533$ & $0.268 \pm 0.067$ & $-0.71 \pm 0.06$ & $-40.2 \pm 0.6$ \\
\hline
\end{tabular}




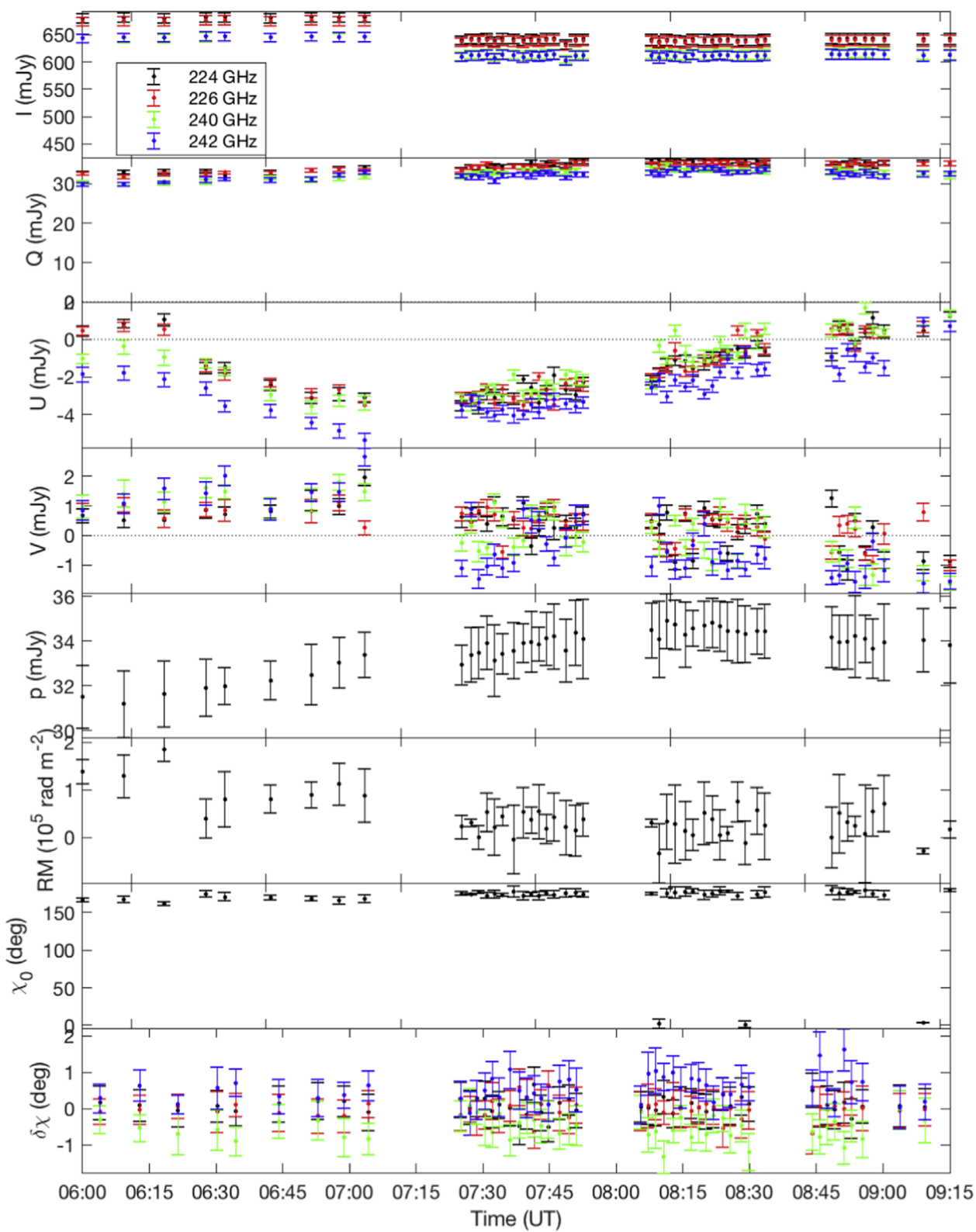

Figure 16. Timeseries plot for J1733-3722 in epoch 2 following Figure 3. 


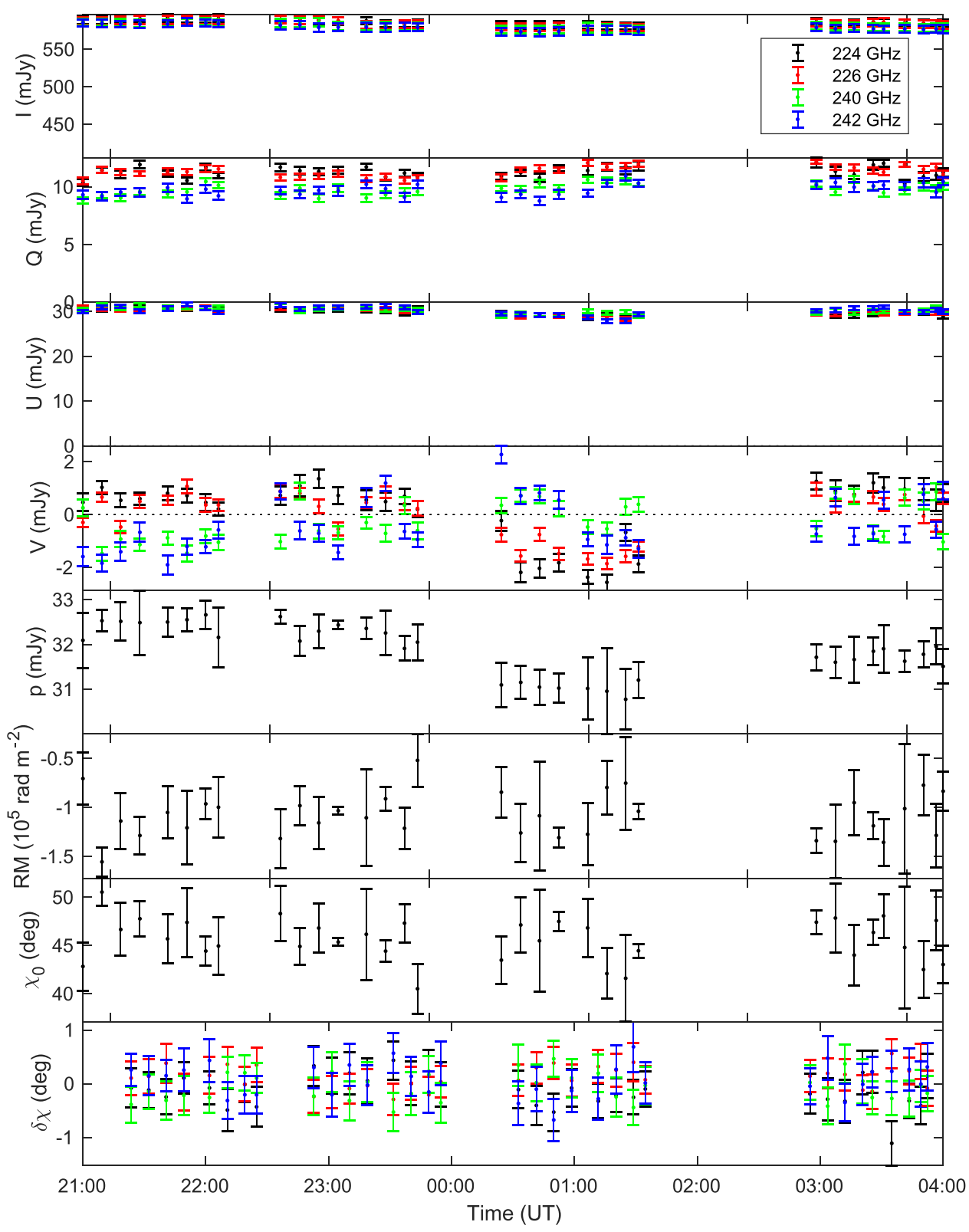

Figure 17. Timeseries plot for J1733-3722 in epoch 3 following Figure 3. 
The Astrophysical Journal, 868:101 (25pp), 2018 December 1

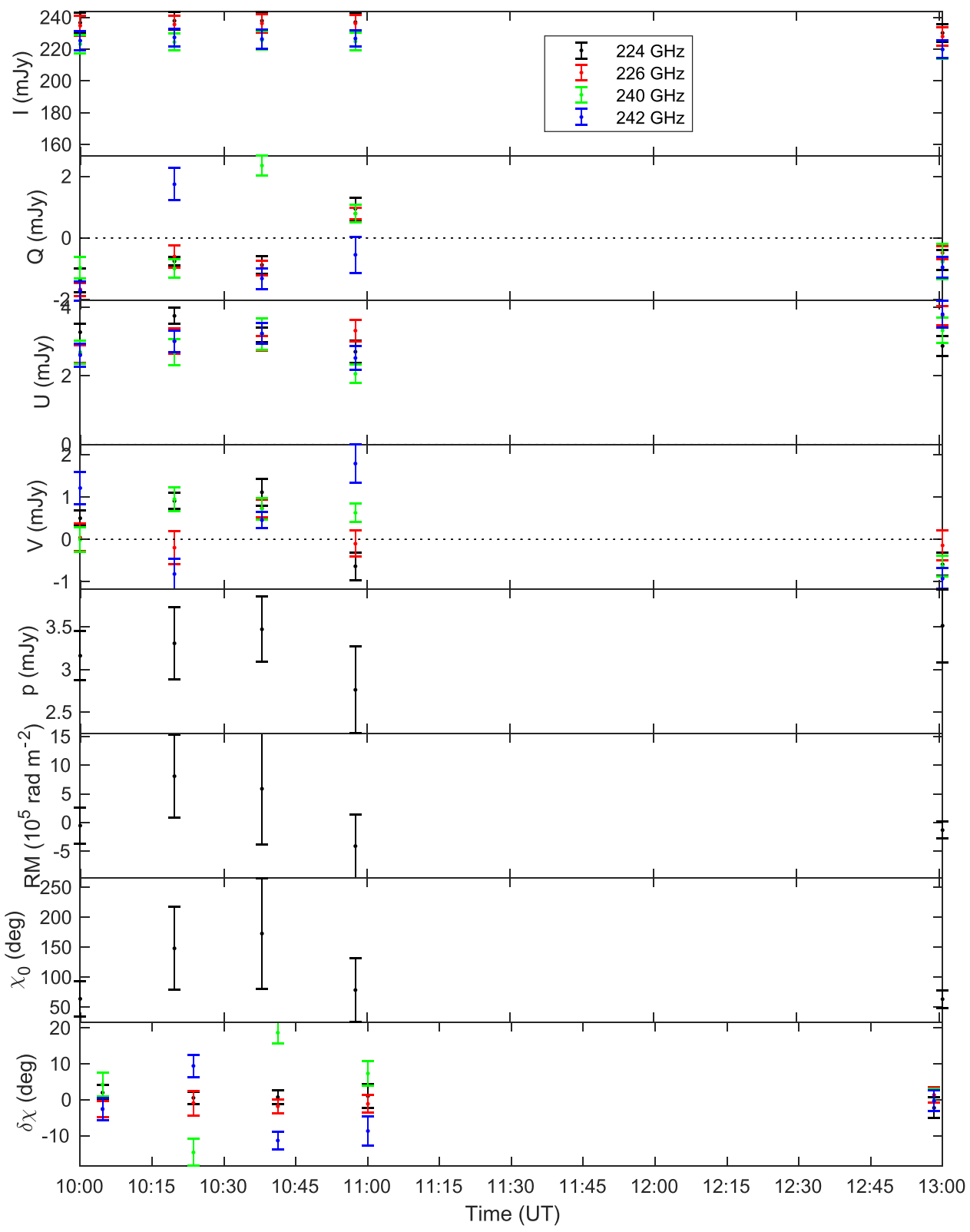

Figure 18. Timeseries plot for J1713-3418 in epoch 1 following Figure 3. 
The Astrophysical Journal, 868:101 (25pp), 2018 December 1

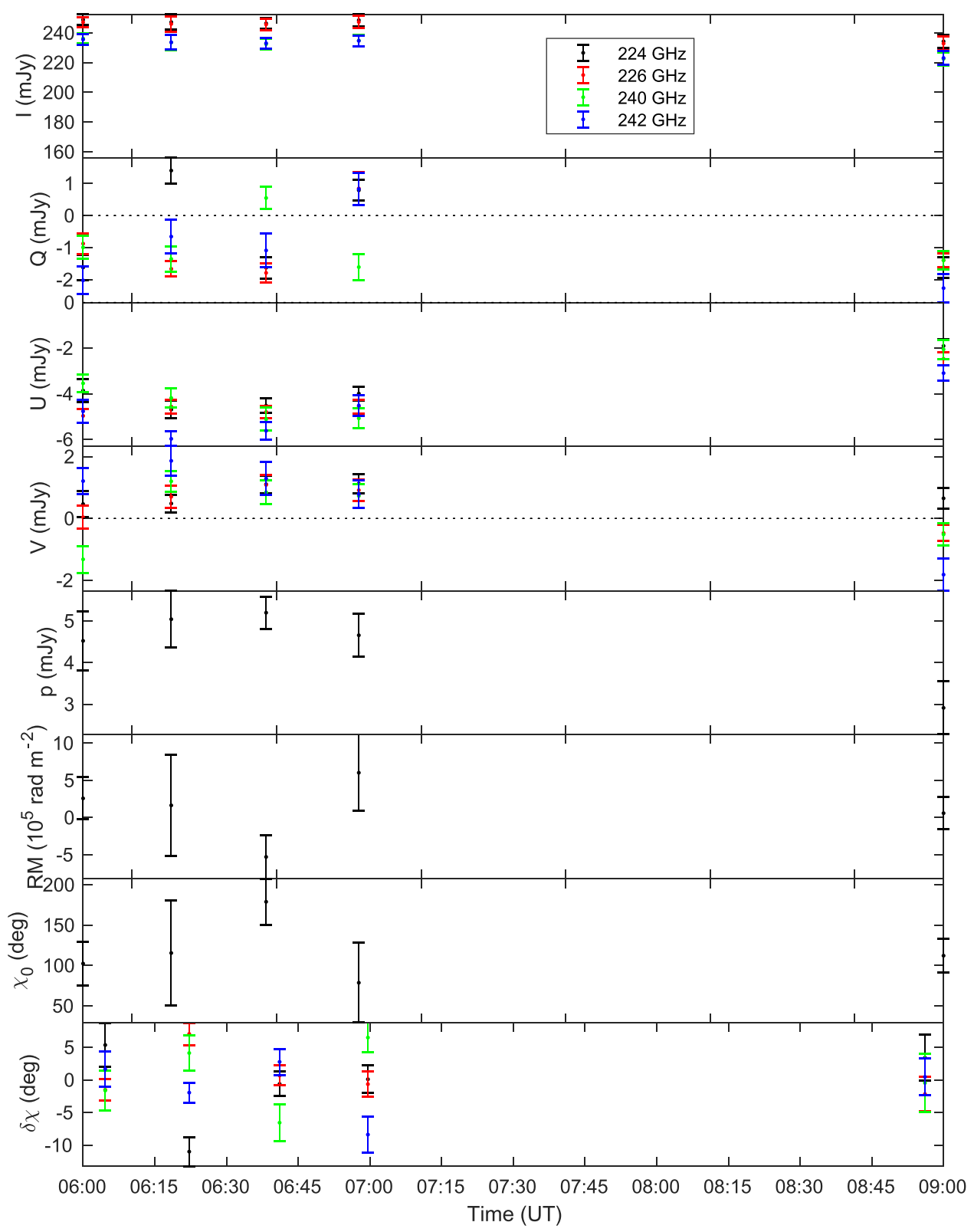

Figure 19. Timeseries plot for J1713-3418 in epoch 2 following Figure 3. 


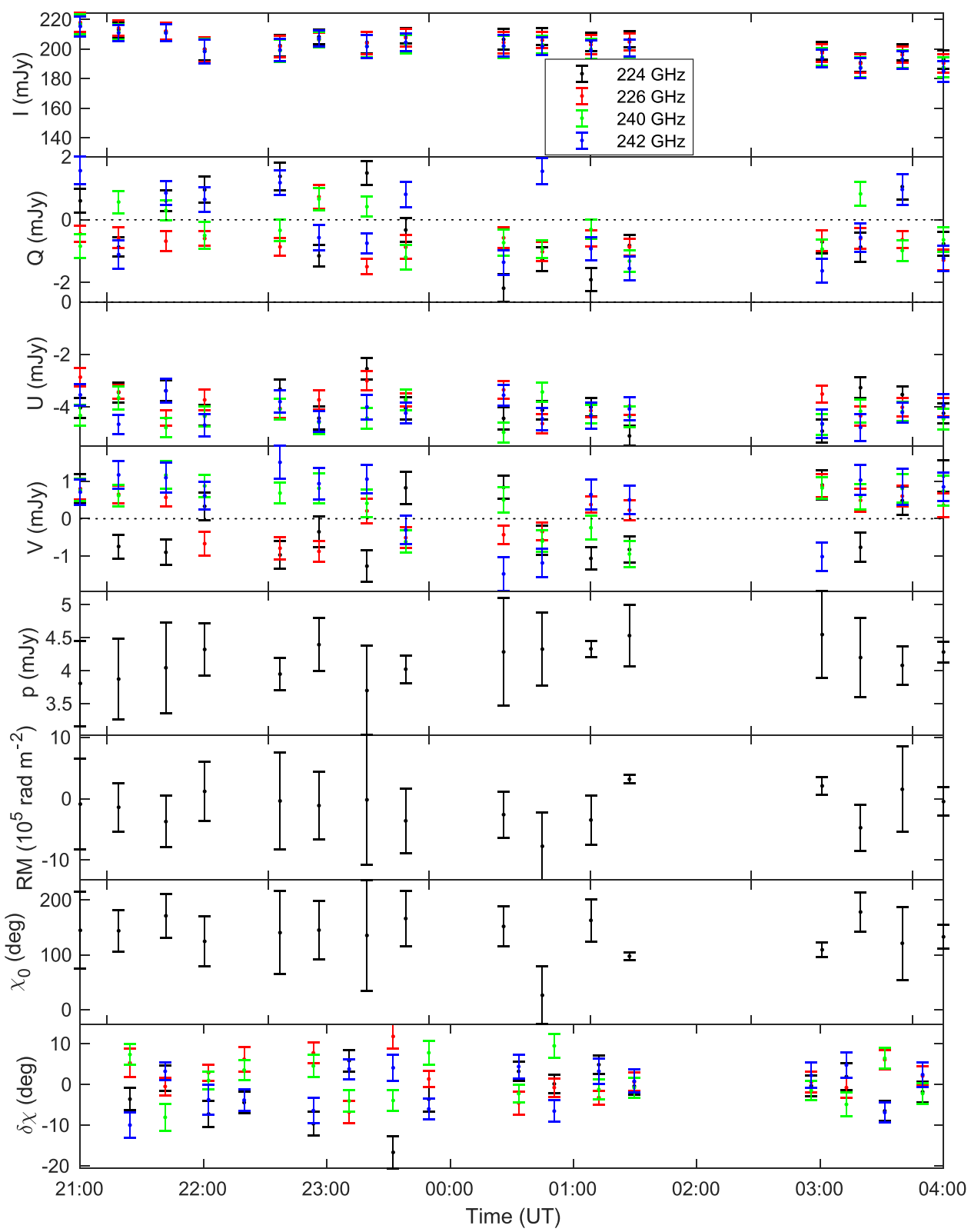

Figure 20. Timeseries plot for J1713-3418 in epoch 3 following Figure 3. 
The Astrophysical Journal, 868:101 (25pp), 2018 December 1

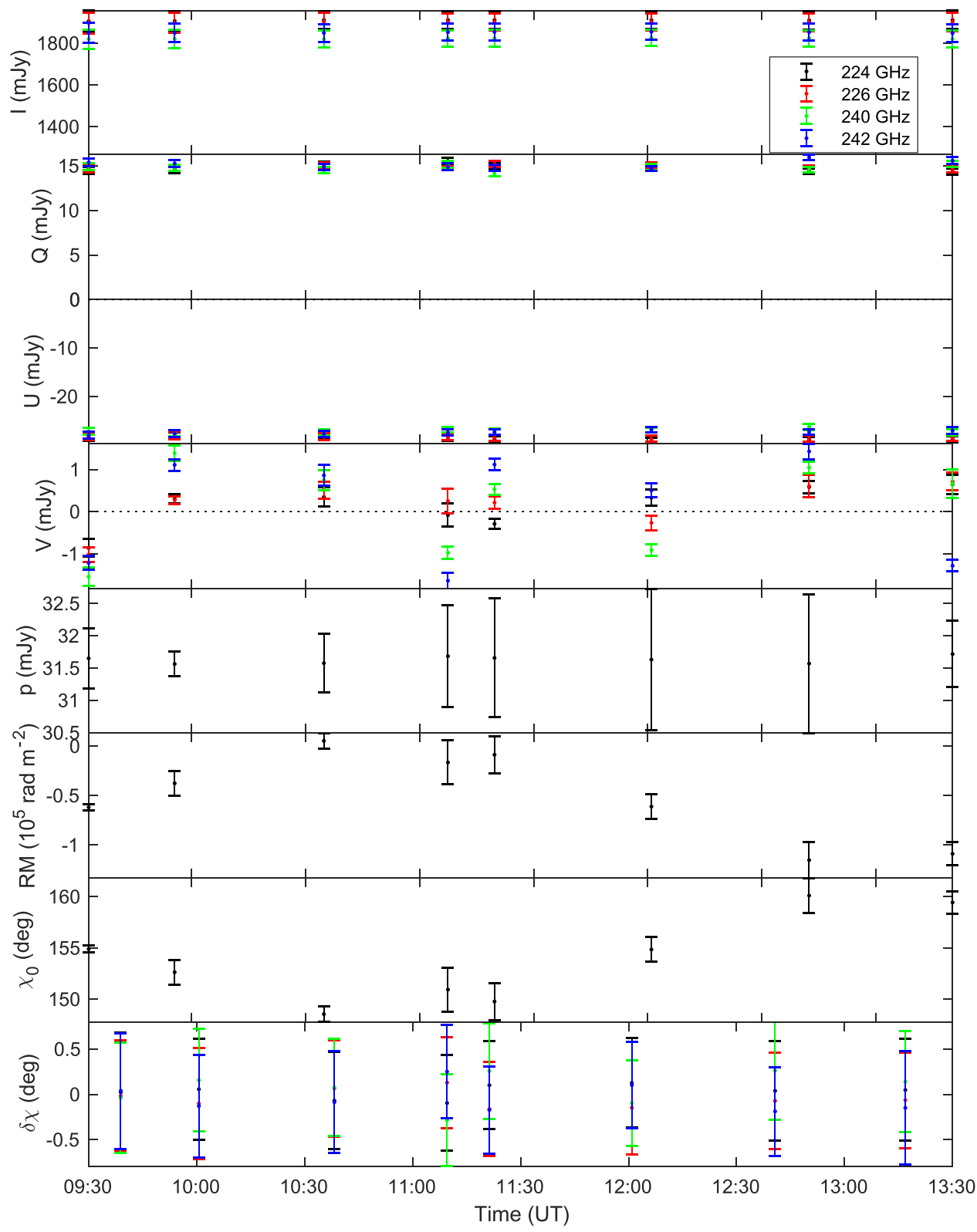

Figure 21. Timeseries plot for J1751+0939 in epoch 1 following Figure 3. 
The Astrophysical Journal, 868:101 (25pp), 2018 December 1

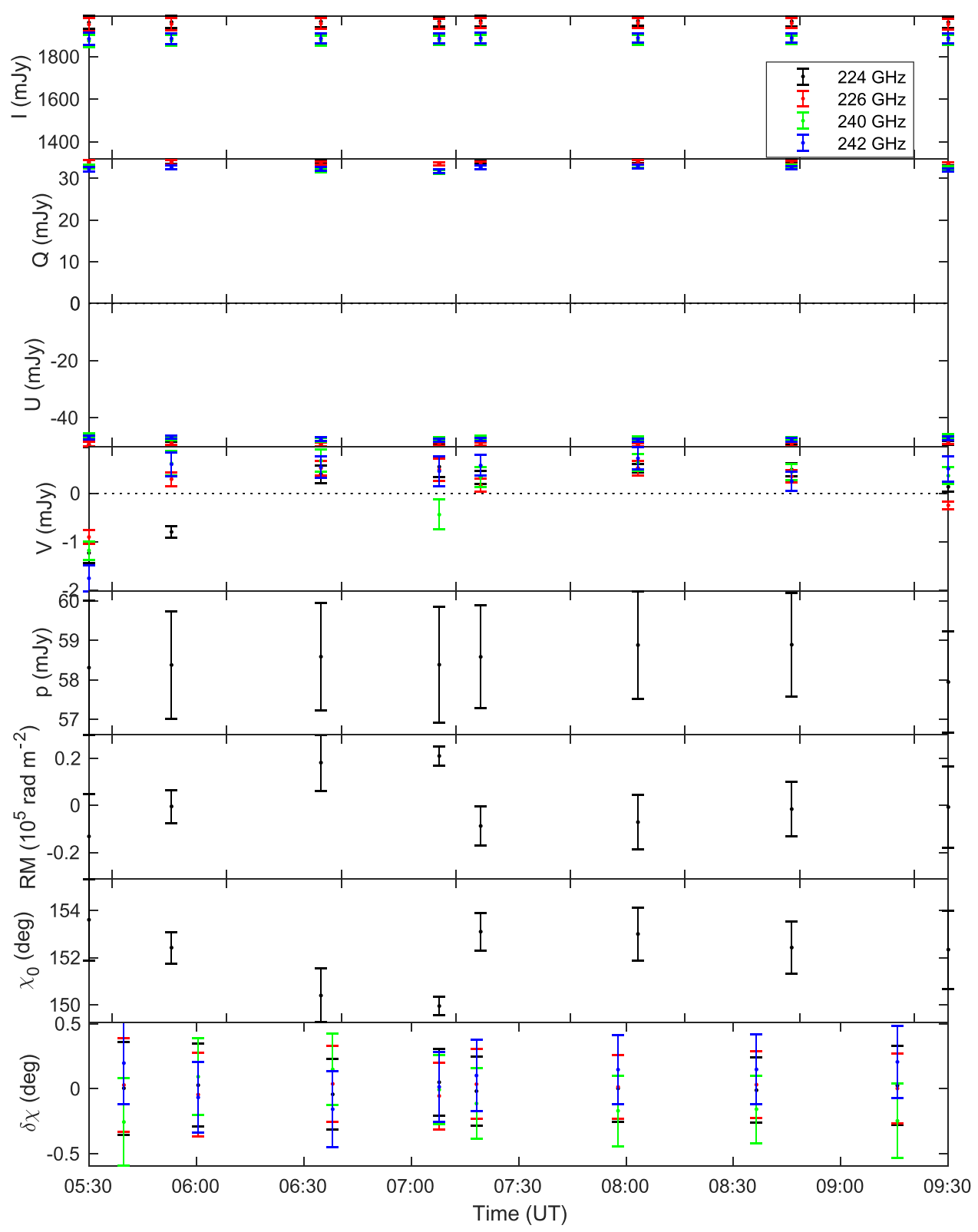

Figure 22. Timeseries plot for J1751+0939 in epoch 2 following Figure 3. 
The Astrophysical Journal, 868:101 (25pp), 2018 December 1

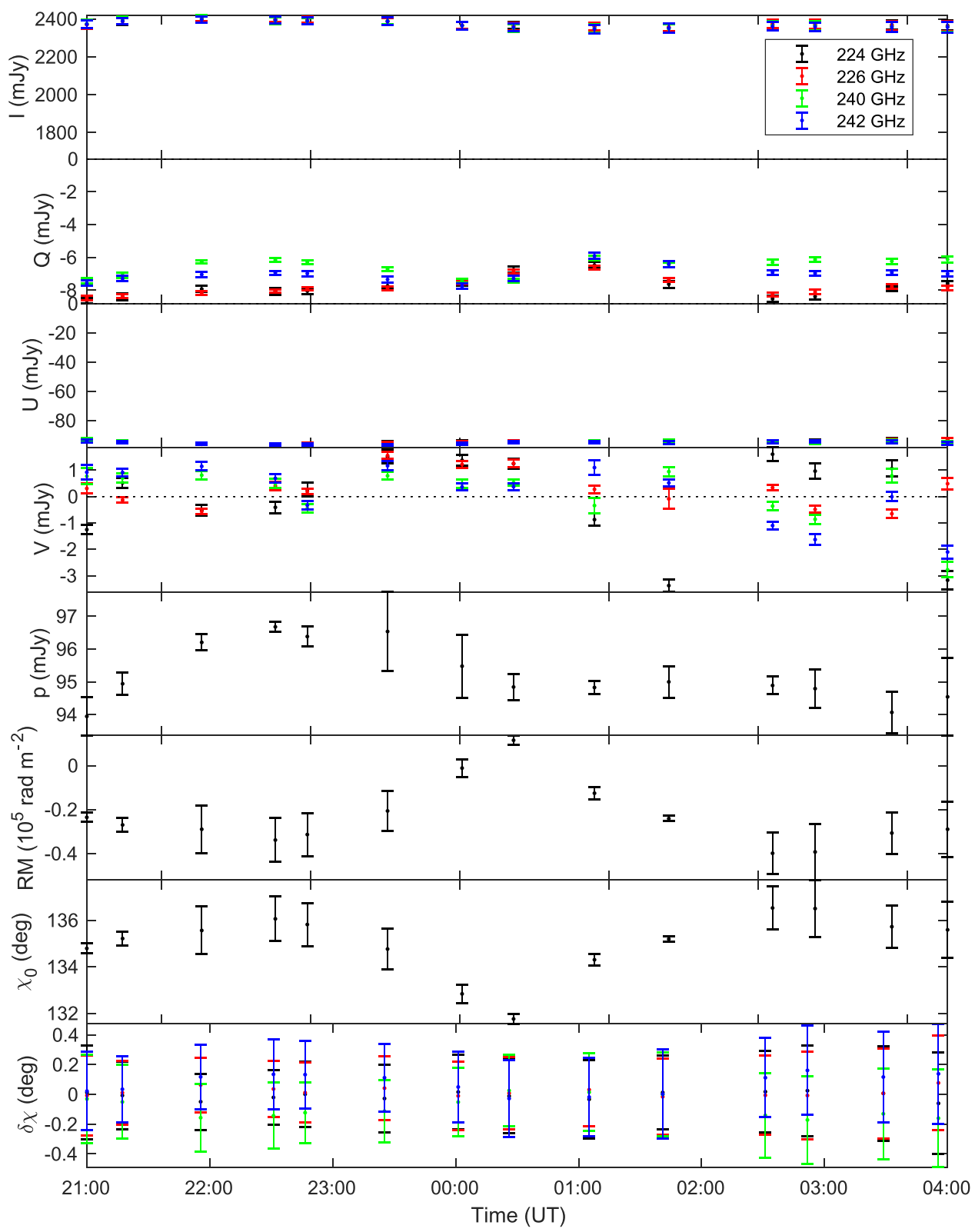

Figure 23. Timeseries plot for J1751+0939 in epoch 3 following Figure 3. 


\section{A.2. Intra-epoch Properties}

As with the epoch-averaged results, the results for the polarization calibrator, $\mathrm{J} 1751+0939$, present the limits of calibration accuracy. In all three epochs, we see very stable measurements in each Stokes parameter as a function of time. Variations in the fitted RM are $\lesssim 3 \times 10^{4} \mathrm{rad} \mathrm{m}^{-2}$ and in the fitted $\bar{\chi} \lesssim 0.5$. Results for the phase calibrator J1733-3722 and for the check source are also stable over the course of each track in the Stokes parameters. The stability of the calibrator results is best seen in Figures 15-23. For J1733-3722, we see a maximum variation in the fitted position angle $\bar{\chi}$ of $\lesssim 4^{\circ}$ and rms variations of $\lesssim 1^{\circ}$. The RM has an rms variation $<10^{4} \mathrm{rad} \mathrm{m}^{-2}$. For $\mathrm{J} 1713-3418$, the variations are slightly larger owing to the lower source flux but consistent with no change in either RM or $\bar{\chi}$. The rms variations in $\bar{\chi}$ are at most $6^{\circ}$, and $\mathrm{RM}$ variations are $\lesssim 10^{5} \mathrm{rad} \mathrm{m}^{-2}$.

\section{ORCID iDs}

Geoffrey C. Bower 나 https://orcid.org/0000-0003-4056-9982 Jason Dexter 누 https://orcid.org/0000-0003-3903-0373 Heino Falcke (1) https://orcid.org/0000-0002-2526-6724 Vincent Fish (ib https://orcid.org/0000-0002-7128-9345 Michael D. Johnson (1) https://orcid.org/0000-0002-4120-3029 Daniel P. Marrone (i) https://orcid.org/0000-0002-2367-1080 Alison Peck (i) https://orcid.org/0000-0001-8276-0000 Richard L. Plambeck iㅣ https://orcid.org/0000-00016765-9609

Ramprasad Rao (1) https://orcid.org/0000-0002-1407-7944

\section{References}

Aitken, D. K., Greaves, J., Chrysostomou, A., et al. 2000, ApJL, 534, L173 Beckert, T., \& Falcke, H. 2002, A\&A, 388, 1106

Boehle, A., Ghez, A. M., Schödel, R., et al. 2016, ApJ, 830, 17

Bower, G. C., Backer, D. C., Zhao, J. H., Goss, M., \& Falcke, H. 1999a, ApJ, 521,582

Bower, G. C., Deller, A., Demorest, P., et al. 2015a, ApJ, 798, 120

Bower, G. C., Dexter, J., Markoff, S., Rao, R., \& Plambeck, R. L. 2017, ApJL, 843, L31

Bower, G. C., Falcke, H., \& Backer, D. C. 1999b, ApJL, 523, L29

Bower, G. C., Falcke, H., Herrnstein, R. M., et al. 2004, Sci, 304, 704

Bower, G. C., Falcke, H., \& Mellon, R. R. 2002a, ApJL, 578, L103

Bower, G. C., Falcke, H., Sault, R. J., \& Backer, D. C. 2002b, ApJ, 571, 843

Bower, G. C., Falcke, H., Wright, M. C., \& Backer, D. C. 2005, ApJL, 618, L29

Bower, G. C., Goss, W. M., Falcke, H., Backer, D. C., \& Lithwick, Y. 2006, ApJL, 648, L127

Bower, G. C., Markoff, S., Brunthaler, A., et al. 2014, ApJ, 790, 1

Bower, G. C., Markoff, S., Dexter, J., et al. 2015b, ApJ, 802, 69

Bower, G. C., Wright, M. C. H., Backer, D. C., \& Falcke, H. 1999c, ApJ, 527, 851

Bower, G. C., Wright, M. C. H., Falcke, H., \& Backer, D. C. 2003, ApJ, 588, 331

Brinkerink, C. D., Müller, C., Falcke, H., et al. 2016, MNRAS, 462, 1382

Broderick, A. E., Johannsen, T., Loeb, A., \& Psaltis, D. 2014, ApJ, 784, 7
Brunthaler, A., Bower, G. C., Falcke, H., \& Mellon, R. R. 2001, ApJL, 560, L123

Davelaar, J., Mościbrodzka, M., Bronzwaer, T., \& Falcke, H. 2018, A\&A, 612, A34

Desvignes, G., Eatough, R. P., Pen, U. L., et al. 2018, ApJL, 852, L12

Doeleman, S., Agol, E., Backer, D., et al. 2009, in Astro2010: The Astronomy and Astrophysics Decadal Survey, 68

Doeleman, S. S., Weintroub, J., Rogers, A. E. E., et al. 2008, Natur, 455, 78

Eatough, R. P., Falcke, H., Karuppusamy, R., et al. 2013, Natur, 501, 391

Emmanoulopoulos, D., McHardy, I. M., \& Uttley, P. 2010, MNRAS, 404, 931

Falcke, H., \& Markoff, S. B. 2013, CQGra, 30, 244003

Falcke, H., Melia, F., \& Agol, E. 2000, ApJL, 528, L13

Fish, V., Alef, W., Anderson, J., et al. 2013, arXiv:1309.3519

Fish, V. L., Doeleman, S. S., Beaudoin, C., et al. 2011, ApJL, 727, L36

Fish, V. L., Johnson, M. D., Doeleman, S. S., et al. 2016, ApJ, 820, 90

Gillessen, S., Genzel, R., Fritz, T. K., et al. 2012, Natur, 481, 51

Gold, R., McKinney, J. C., Johnson, M. D., \& Doeleman, S. S. 2017, ApJ, 837,180

Gravity Collaboration, Abuter, R., Amorim, A., et al. 2018, A\&A, 615, L15

Haggard, D., Baganoff, F. K., Ponti, G., et al. 2014, AAS Meeting 14, 100.04

Hovatta, T., O'Sullivan, S., Martí-Vidal, I., Savolainen, T., \& Tchekhovskoy, A. 2018, arXiv:1803.09982

Huang, L., Liu, S., Shen, Z.-Q., et al. 2008, ApJL, 676, L119

Jiménez-Rosales, A., \& Dexter, J. 2018, MNRAS, 478, 1875

Johnson, M. D., Fish, V. L., Doeleman, S. S., et al. 2015, Sci, 350, 1242

Kuo, C. Y., Asada, K., Rao, R., et al. 2014, ApJL, 783, L33

Liu, H. B., Wright, M. C. H., Zhao, J.-H., et al. 2016a, A\&A, 593, A107

Liu, H. B., Wright, M. C. H., Zhao, J.-H., et al. 2016b, A\&A, 593, A44

Lu, R.-S., Krichbaum, T. P., Roy, A. L., et al. 2018, ApJ, 859, 60

Macquart, J.-P., Bower, G. C., Wright, M. C. H., Backer, D. C., \& Falcke, H. 2006, ApJL, 646, L111

Marrone, D. P., Moran, J. M., Zhao, J.-H., \& Rao, R. 2006, JPhCS, 54, 354

Marrone, D. P., Moran, J. M., Zhao, J.-H., \& Rao, R. 2007, ApJL, 654, L57

Mościbrodzka, M., Dexter, J., Davelaar, J., \& Falcke, H. 2017, MNRAS, 468, 2214

Mościbrodzka, M., Falcke, H., Shiokawa, H., \& Gammie, C. F. 2014, A\&A, 570, A7

Mościbrodzka, M., \& Gammie, C. F. 2018, MNRAS, 475, 43

Mościbrodzka, M., Shiokawa, H., Gammie, C. F., \& Dolence, J. C. 2012, ApJL, 752, L1

Muñoz, D. J., Marrone, D. P., Moran, J. M., \& Rao, R. 2012, ApJ, 745, 115

Nagai, H., Nakanishi, K., Paladino, R., et al. 2016, ApJ, 824, 132

Ortiz-León, G. N., Johnson, M. D., Doeleman, S. S., et al. 2016, ApJ, 824, 40

Pacholczyk, A. G. 1977, Int. Ser. in Natural Philosophy, Vol. 89 (Oxford: Pergamon Press, Ltd.)

Pang, B., Pen, U.-L., Matzner, C. D., Green, S. R., \& Liebendörfer, M. 2011, MNRAS, 415, 1228

Plambeck, R. L., Bower, G. C., Rao, R., et al. 2014, ApJ, 797, 66

Plewa, P. M., Gillessen, S., Pfuhl, O., et al. 2017, ApJ, 840, 50

Quataert, E., Narayan, R., \& Reid, M. J. 1999, ApJL, 517, L101

Ressler, S. M., Tchekhovskoy, A., Quataert, E., \& Gammie, C. F. 2017, MNRAS, 467, 3604

Ruszkowski, M., \& Begelman, M. C. 2002, ApJ, 573, 485

Sault, R. J., \& Macquart, J.-P. 1999, ApJL, 526, L85

Sharma, P., Quataert, E., \& Stone, J. M. 2007, ApJ, 671, 1696

Shcherbakov, R. V., Penna, R. F., \& McKinney, J. C. 2012, ApJ, 755, 133

Shen, Z.-Q., Lo, K. Y., Liang, M.-C., Ho, P. T. P., \& Zhao, J.-H. 2005, Natur, 438,62

Trippe, S., Neri, R., Krips, M., et al. 2012, A\&A, 540, A74

Waisberg, I., Dexter, J., Gillessen, S., et al. 2018, MNRAS, arXiv:1802.08198

Wang, Q. D., Nowak, M. A., Markoff, S. B., et al. 2013, Sci, 341, 981

Witzel, G., Sitarski, B. N., Ghez, A. M., et al. 2017, ApJ, 847, 80

Yuan, F., \& Narayan, R. 2014, ARA\&A, 52, 529 